\title{
NONLINEAR PERTURBATIONS OF A PERIODIC MAGNETIC CHOQUARD EQUATION WITH HARDY-LITTLEWOOD-SOBOLEV CRITICAL EXPONENT
}

\author{
H. BUENO, N. H. LISBOA AND L. L. VIEIRA
}

ABStRACT. In this paper, we consider the following magnetic nonlinear Choquard equation

$$
-(\nabla+i A(x))^{2} u+V(x) u=\left(\frac{1}{|x|^{\alpha}} *|u|^{2_{\alpha}^{*}}\right)|u|^{2_{\alpha}^{*}-2} u+\lambda f(u) \text { in } \mathbb{R}^{N},
$$

where $2_{\alpha}^{*}=\frac{2 N-\alpha}{N-2}$ is the critical exponent in the sense of the Hardy-Littlewood-Sobolev inequality, $\lambda>0, N \geq 3$, $0<\alpha<N, A: \mathbb{R}^{N} \rightarrow \mathbb{R}^{N}$ is an $C^{1}, \mathbb{Z}^{N}$-periodic vector potential and $V$ is a continuous scalar potential given as a perturbation of a periodic potential. Under suitable assumptions on different types of nonlinearities $f$, namely, $f(x, u)=\left(\frac{1}{|x|^{\alpha}} *|u|^{p}\right)|u|^{p-2} u$ for $(2 N-\alpha) / N<p<2_{\alpha}^{*}$, then $f(u)=|u|^{p-1} u$ for $1<p<2^{*}-1$ and $f(u)=|u|^{2^{*}-2} u$ (where $2^{*}=2 N /(N-2)$ ), we prove the existence of at least one ground state solution for this equation by variational methods if $p$ belongs to some intervals depending on $N$ and $\lambda$.

Keywords: Variational methods, magnetic Choquard equation, Hardy-Littlewood-Sobolev critical exponent

MSC[2010]: 35Q55, 35Q40, 35J20

\section{INTRODUCTION}

In this article we consider the problem

$$
-(\nabla+i A(x))^{2} u+V(x) u=\left(\frac{1}{|x|^{\alpha}} *|u|^{2_{\alpha}^{*}}\right)|u|^{2_{\alpha}^{*}-2} u+\lambda f(u) \text { in } \mathbb{R}^{N},
$$

where $\nabla+i A(x)$ is the covariant derivative with respect to the $C^{1}, \mathbb{Z}^{N}$-periodic vector potential $A: \mathbb{R}^{N} \rightarrow \mathbb{R}^{N}$, i.e,

$$
A(x+y)=A(x), \forall x \in \mathbb{R}^{N}, \forall y \in \mathbb{Z}^{N} .
$$

The exponent $2_{\alpha}^{*}=\frac{2 N-\alpha}{N-2}$ is critical, in the sense of the Hardy-Littlewood-Sobolev inequality, $\lambda>0, N \geq 3$, $0<\alpha<N, V: \mathbb{R}^{N} \rightarrow \mathbb{R}$ is a continuous scalar potential and $f$ stands for different types of nonlinearities. Namely, we first consider $f(x, u)=\left(\frac{1}{|x|^{\alpha}} *|u|^{p}\right)|u|^{p-2} u$ for $(2 N-\alpha) / N<p<2_{\alpha}^{*}$, then $f(u)=|u|^{p-1} u$ for $1<p<2^{*}-1$, where $2^{*}$ is the critical exponent of immersion $D^{1,2}\left(\mathbb{R}^{N}\right) \hookrightarrow L^{2^{*}}\left(\mathbb{R}^{N}\right)$, and finally we examine $f(u)=|u|^{2^{*}-2} u$.

Inspired by the seminal work of Coti Zelati and Rabinowitz [14, but also by Alves, Carrião and Miyagaki [1] and by Alves and Figueiredo [2, we assume that there is a continuous, $\mathbb{Z}^{N}$-periodic potential $V_{\mathcal{P}}: \mathbb{R}^{N} \rightarrow \mathbb{R}$, constants $V_{0}, W_{0}>0$ and $W \in L^{\frac{N}{2}}\left(\mathbb{R}^{N}\right)$ with $W(x) \geq 0$ such that

$\left(V_{1}\right) V_{\mathcal{P}}(x) \geq V_{0}, \quad \forall x \in \mathbb{R}^{N}$

$\left(V_{2}\right) V(x)=V_{\mathcal{P}}(x)-W(x) \geq W_{0}, \quad \forall x \in \mathbb{R}^{N}$,

where the last inequality is strict on a subset of positive measure in $\mathbb{R}^{N}$.

Since the problem is considered in the whole $\mathbb{R}^{N}$ and has a critical nonlinearity in the Hardy-Littlewood-Sobolev sense, the verification of any compactness condition is not easy.

Our paper is motivated by Gao and Yang in [20, where a classical Choquard equation is considered in a bounded domain, i.e., the case $A \equiv 0$ and $V \equiv 0$ is studied in a bounded domain $\Omega$. There is a huge literature about the Choquard equation and we cite only Moroz and Van Schaftingen [27] for a good review of results on this important subject. In [20, Gao and Yang proved the existence of a ground state solution under restriction on $N$ and $\lambda$. Other recent advances in the study of the Choquard equation can be found, e.g., in [4, 5, 6, 17, 18, 22, 26, 30. 
In Mukherjee and Sreenadh [28, the magnetic problem

$$
-(\nabla+i A(x))^{2} u+\mu g(x) u=\lambda u+\left(\frac{1}{|x|^{\alpha}} *|u|^{2_{\alpha}^{*}}\right)|u|^{2_{\alpha}^{*}-2} u \text { in } \mathbb{R}^{N}
$$

was examined. In this equation $\mu>0$ is also a parameter that interacts with the linear term in the right-hand side of the equation. Under suitable hypotheses on $g$, the existence of a ground state solution was proved. The concentration of solutions as $\mu \rightarrow \infty$ was also studied.

Changing the right-hand side of (1) to

$$
\left.\left(\frac{1}{|x|^{\alpha}} *|u|^{p}\right)\right)|u|^{p-2} u
$$

the problem was studied by Cingolani, Clapp and Secchi in 13. In that paper the authors proved existence and multiplicity of solutions. In [12, the right-hand side (2) was generalized and a ground state solution was obtained, but the multiplicity result depend on more restrictive hypotheses than in [13].

Recent years have witnessed a growth of interest in the study of magnetic equations. The progress in this research can be found in a series of articles, e.g., [3, 7, 8, 9, 15, 16].

The main results of this paper are the following theorems.

Theorem 1. For $\frac{2 N-\alpha}{N}<p<2_{\alpha}^{*}$, under the hypotheses already stated on $A, V$ and $\alpha$, problem

$$
\left.\left.-(\nabla+i A(x))^{2} u+V(x) u=\left(\frac{1}{|x|^{\alpha}} *|u|^{2_{\alpha}^{*}}\right)\right)|u|^{2_{\alpha}^{*}-2} u+\lambda\left(\frac{1}{|x|^{\alpha}} *|u|^{p}\right)\right)|u|^{p-2} u \text { in } \mathbb{R}^{N}
$$

has at least one ground state solution if either

(i) $\frac{N+2-\alpha}{N-2}<p<2_{\alpha}^{*}, N=3,4$ and $\lambda>0$;

(ii) $\frac{2 N-\alpha}{N}<p \leq \frac{N+2-\alpha}{N-2}, N=3,4$ and $\lambda$ sufficiently large;

(iii) $\frac{2 N-2-\alpha}{N-2}<p<2_{\alpha}^{*}, N \geq 5$ and $\lambda>0$;

(iv) $\frac{2 N-\alpha}{N}<p \leq \frac{2 N-2-\alpha}{N-2}, N \geq 5$ and $\lambda$ sufficiently large.

Theorem 2. For $1<p<2^{*}-1$, under the hypotheses already stated on $A, V$ and $\alpha$, problem

$$
-(\nabla+i A(x))^{2} u+V(x) u=\left(\frac{1}{|x|^{\alpha}} *|u|^{2_{\alpha}^{*}}\right)|u|^{2_{\alpha}^{*}-2} u+\lambda|u|^{p-1} u \text { in } \mathbb{R}^{N} .
$$

has at least one ground state solution if either

(i) $3<p<5, N=3$ and $\lambda>0$;

(ii) $p>1, N \geq 4$ and $\lambda>0$;

(iii) $1<p \leq 3, N=3$ and $\lambda$ sufficiently large.

Theorem 3. Under the hypotheses already stated on $A, V$ and $\alpha$, the problem

$$
-(\nabla+i A(x))^{2} u+V(x) u=\lambda\left(\frac{1}{|x|^{\alpha}} *|u|^{p}\right)|u|^{p-2} u+|u|^{2^{*}-2} u \text { in } \mathbb{R}^{N},
$$

has at least one ground state solution in the intervals already described in Theorem 1.

Initially, we are going to prove the existence of a ground state solution for problem (11) considering the potential $V=V_{\mathcal{P}}$, that is, we consider the problem

$$
\left.-(\nabla+i A(x))^{2} u+V_{\mathcal{P}}(x) u=\left(\frac{1}{|x|^{\alpha}} *|u|^{2_{\alpha}^{*}}\right)\right)|u|^{2_{\alpha}^{*}-2} u+\lambda f(u) \text { in } \mathbb{R}^{N}
$$

and $f$ as in Theorems 11, 2 and 3. where we maintain the notation introduced before and suppose that $\left(V_{1}\right)$ is valid.

As in Gao and Yang in [20, the key step to proof the existence of a ground state solution of problem (6) is the use of cut-off techniques on the extreme function that attains the best constant $S_{H, L}$ defined in the sequence. This allows us to estimate the mountain pass value $c_{\lambda}$ associated with the energy functional $J_{A, V_{\mathcal{P}}}$ related with (6) in terms of the level where the PS condition holds. In a demanding proof, this lead us to establish intervals for $p$ (depending on $N$ and $\lambda$ ) where the PS condition is satisfied, as in the seminal work of Brézis and Nirenberg 
[11. After that, the proof is completed by showing the mountain pass geometry, introducing the Nehari manifold associated with (6) and applying concentration-compactness arguments. In the sequel, we consider (10) for the different nonlinearities $f$ and prove that each problem has at least one ground state solution.

We observe that the conclusion of Theorem 2 is similar to that of Theorem 1.1 in Alves, Carrião and Miyagaki [1] and Theorem 1.1 in Miyagaki 25]. Being more precise, in [1] the authors have discussed the existence of a positive solution to the semilinear elliptic problem involving critical exponents

$$
-\Delta u+V(x) u=\lambda u^{q}+u^{p} \text { in } \mathbb{R}^{N},
$$

where $\lambda>0$ is a parameter, $1<q<p=2^{*}-1$ and $V: \mathbb{R}^{N} \rightarrow \mathbb{R}$ is a positive continuous function. On its turn, Miyagaki 25] has studied the existence of nontrivial solution for the following class of semilinear elliptic equation in $\mathbb{R}^{N}(N \geq 3)$ involving critical Sobolev exponents

$$
-\Delta u+a(x) u=\lambda|u|^{q-1}+|u|^{p-1} u \text { in } \mathbb{R}^{N},
$$

where $1<q<p \leq 2^{*}-1=\frac{N+2}{N-2}$ and $\lambda>0$ are constants and $a: \mathbb{R}^{N} \rightarrow \mathbb{R}$ is a continuous function such that $a(x) \geq a_{0}$ for all $x \in \mathbb{R}^{N}$, where $a_{0}>0$ is a constant.

Problems (60) and (10) are then related by showing that the minimax value $d_{\lambda}$ of the latter satisfies $d_{\lambda}<c_{\lambda}$. Once more, concentration-compactness arguments are applied to show the existence of a ground state solution.

This paper is organized as follows. In Section 2 some preliminary results will be established. Section 3,4 and 5 are then devoted to the proofs of Theorems 1, 2 and 3, respectively.

\section{Preliminary Results}

We denote

We handle problem (10) in the space

$$
\nabla_{A} u=\nabla u+i A(x) u
$$

$$
H_{A, V}^{1}\left(\mathbb{R}^{N}, \mathbb{C}\right)=\left\{u \in L^{2}\left(\mathbb{R}^{N}, \mathbb{C}\right): \nabla_{A} u \in L^{2}\left(\mathbb{R}^{N}, \mathbb{C}\right), \quad \int_{\mathbb{R}^{N}} V(x)|u(x)|^{2} \mathrm{~d} x<\infty\right\}
$$

endowed with the norm

$$
\|u\|_{A, V}=\left(\int_{\mathbb{R}^{N}}\left(\left|\nabla_{A} u\right|^{2}+V(x)|u|^{2}\right) \mathrm{d} x\right)^{\frac{1}{2}}
$$

Observe that the norm generated by this scalar product is equivalent to the norm obtained by considering $V \equiv 1$, see [24, Definition 7.20].

If $u \in H_{A, V}^{1}\left(\mathbb{R}^{N}, \mathbb{C}\right)$, then $|u| \in H^{1}\left(\mathbb{R}^{N}\right)$ and the diamagnetic inequality is valid (see [13] or [24, Theorem 7.21])

$$
|\nabla| u|(x)| \leq|\nabla u(x)+i A(x) u(x)| \text {, a.e. } x \in \mathbb{R}^{N} .
$$

As a consequence of the diamagnetic inequality, we have the continuous immersion

$$
H_{A, V}^{1}\left(\mathbb{R}^{N}, \mathbb{C}\right) \hookrightarrow L^{s}\left(\mathbb{R}^{N}, \mathbb{C}\right)
$$

for any $s \in\left[2, \frac{2 N}{N-2}\right]$. We denote $2^{*}=\frac{2 N}{N-2}$ and $\|\cdot\|_{s}$ the norm in $L^{s}\left(\mathbb{R}^{N}, \mathbb{C}\right)$.

It is well-known that $C_{c}^{\infty}\left(\mathbb{R}^{N}, \mathbb{C}\right)$ is dense in $H_{A, V_{\mathcal{P}}}^{1}\left(\mathbb{R}^{N}, \mathbb{C}\right)$, see [24, Theorem 7.22].

Following Gao and Yang [21, we denote by $S_{H, L}$

$$
\begin{aligned}
S_{H, L}: & =\inf _{u \in D^{1,2}\left(\mathbb{R}^{N}, \mathbb{R}\right) \backslash\{0\}} \frac{\int_{\mathbb{R}^{N}}|\nabla u|^{2} \mathrm{~d} x}{\left(\int_{\mathbb{R}^{N}} \int_{\mathbb{R}^{N}} \frac{\left|u(x)^{2_{\alpha}^{*}}\right||u(y)|^{2_{\alpha}^{*}}}{|x-y|^{\alpha}} \mathrm{d} x \mathrm{~d} y\right)^{\frac{N-2}{2 N-\alpha}}} \\
& =\inf _{u \in D_{A}^{1,2}\left(\mathbb{R}^{N}\right) \backslash\{0\}} \frac{\int_{\mathbb{R}^{N}}\left|\nabla_{A} u\right|^{2} \mathrm{~d} x}{\left(\int_{\mathbb{R}^{N}} \int_{\mathbb{R}^{N}} \frac{\left|u(x)^{2_{\alpha}^{*}}\right||u(y)|^{2_{\alpha}^{*}}}{|x-y|^{\alpha}} \mathrm{d} x \mathrm{~d} y\right)^{\frac{N-2}{2 N-\alpha}}}=: S_{A},
\end{aligned}
$$


where $\left.\left.D_{A}^{1,2}\left(\mathbb{R}^{N}\right)=\left\{u \in L^{2^{*}}\left(\mathbb{R}^{N}, \mathbb{C}\right)\right\}\right): \nabla_{A} u \in L^{2}\left(\mathbb{R}^{N}, \mathbb{C}\right)\right\}$. The equality between $S_{H, L}$ and $S_{A}$ was proved in Mukherjee and Sreenadh [28. We remark that $S_{A}$ is attained if and only if $\operatorname{rot} A=0$ [28, Theorem 4.1]. See also 10, Theorem 1.1].

We state a result proved in 21].

Proposition 4 (Gao and Yang [21). The constant $S_{H, L}$ defined in (8) is achieved if and only if

$$
u=C\left(\frac{b}{b^{2}+|x-a|^{2}}\right)^{\frac{N-2}{2}},
$$

where $C>0$ is a fixed constant, $a \in \mathbb{R}^{N}$ and $b \in(0, \infty)$ are parameters. Furthermore,

$$
S_{H, L}=\frac{S}{C(N, \alpha)^{\frac{N-2}{2 N-\alpha}}},
$$

where $S$ is the best Sobolev constant of the immersion $D^{1,2}\left(\mathbb{R}^{N}\right) \hookrightarrow L^{2^{*}}\left(\mathbb{R}^{N}\right)$ and $C(N, \alpha)$ depends on $N$ and $\alpha$.

If we consider the minimizer for $S$ given by $U(x):=\frac{[N(N-2)]^{\frac{N-2}{4}}}{\left(\left.|1+| x\right|^{2} \mid\right)^{\frac{N-2}{2}}}($ see $[31$, Theorem 1.42]), then

$$
\bar{U}(x)=S^{\frac{(N-\alpha)(2-\alpha)}{4(N+2-\alpha)}} C(N, \alpha)^{\frac{2-N}{2(N+2-\alpha)}} \frac{[N(N-2)]^{\frac{N-2}{4}}}{\left(\left.|1+| x\right|^{2} \mid\right)^{\frac{N-2}{2}}}
$$

is the unique minimizer for $S_{H, L}$ that satisfies

$$
-\triangle u=\left(\int_{\mathbb{R}^{N}} \frac{|u|^{2_{\alpha}^{*}}}{|x-y|^{\alpha}} \mathrm{d} y\right)|u|^{2_{\alpha}^{*}-2} u \text { em } \mathbb{R}^{N}
$$

with

$$
\int_{\mathbb{R}^{N}}|\nabla \bar{U}|^{2} \mathrm{~d} x=\int_{\mathbb{R}^{N}} \int_{\mathbb{R}^{N}} \frac{|\bar{U}(x)|^{2_{\alpha}^{*}}|\bar{U}(y)|^{2_{\alpha}^{*}}}{|x-y|^{\alpha}} \mathrm{d} x \mathrm{~d} y=S_{H, L}^{\frac{2 N-\alpha}{N+2-\alpha}} .
$$

Proposition 5 (Hardy-Littlewood-Sobolev inequality, see [24). Suppose that $f \in L^{t}\left(\mathbb{R}^{N}\right)$ and $h \in L^{r}\left(\mathbb{R}^{N}\right)$ for $t, r>1$ and $0<\alpha<N$ satisfying $\frac{1}{t}+\frac{\alpha}{N}+\frac{1}{r}=2$. Then, there exists a sharp constant $C(t, N, \alpha, r)$, independent of $f$ and $h$, such that

$$
\int_{\mathbb{R}^{N}} \int_{\mathbb{R}^{N}} \frac{f(x) h(y)}{|x-y|^{\alpha}} \mathrm{d} x \mathrm{~d} y \leq C(t, N, \alpha, r)\|f\|_{t}\|h\|_{r} .
$$

If $t=r=\frac{2 N}{2 N-\alpha}$, then

$$
C(t, N, \alpha, r)=C(N, \alpha)=\pi^{\frac{\alpha}{2}} \frac{\Gamma\left(\frac{N}{2}-\frac{\alpha}{2}\right)}{\Gamma\left(N-\frac{\alpha}{2}\right)}\left\{\frac{\Gamma\left(\frac{N}{2}\right)}{\Gamma(N)}\right\}^{-1+\frac{\alpha}{N}} .
$$

In this case there is equality in (9) if and only if $h=c f$ for a constant $c$ and

$$
f(x)=A\left(\gamma^{2}+|x-a|^{2}\right)^{-(2 N-\alpha) / 2}
$$

for some $A \in \mathbb{C}, 0 \neq \gamma \in \mathbb{R}$ and $a \in \mathbb{R}^{N}$.

Lemma 6. Let $U \subseteq \mathbb{R}^{N}$ be any open set. For $1<p<\infty$, let $\left(f_{n}\right)$ be a bounded sequence in $L^{s}(U, \mathbb{C})$ such that $f_{n}(x) \rightarrow f(x)$ a.e. Then $f_{n} \rightarrow f$ in $L^{s}(U, \mathbb{C})$.

The proof of Lemma [ only adapts the arguments given for the real case, as in [23, Lemme 4.8, Chapitre 1]. 
3. THE CASE $f(x, u)=\left(\frac{1}{|x|^{\alpha}} *|u|^{p}\right)|u|^{p-2} u$

3.1. The periodic problem. In this subsection we deal with problem (6) for $f(x, u)$ as above, that is,

$$
\left.\left.-(\nabla+i A(x))^{2} u+V_{\mathcal{P}}(x) u=\left(\frac{1}{|x|^{\alpha}} *|u|^{2_{\alpha}^{*}}\right)\right)|u|^{2_{\alpha}^{*}-2} u+\lambda\left(\frac{1}{|x|^{\alpha}} *|u|^{p}\right)\right)|u|^{p-2} u,
$$

where $\frac{2 N-\alpha}{N}<p<2_{\alpha}^{*}$.

We consider the space

$$
H_{A, V_{\mathcal{P}}}^{1}\left(\mathbb{R}^{N}, \mathbb{C}\right)=\left\{u \in L^{2}\left(\mathbb{R}^{N}, \mathbb{C}\right): \nabla_{A} u \in L^{2}\left(\mathbb{R}^{N}, \mathbb{C}\right)\right\}
$$

endowed with scalar product

$$
\langle u, v\rangle_{A, V_{\mathcal{P}}}=\mathfrak{R e} \int_{\mathbb{R}^{N}}\left(\nabla_{A} u \cdot \overline{\nabla_{A} v}+V_{\mathcal{P}}(x) u \bar{v}\right) \mathrm{d} x
$$

and, therefore

$$
\|u\|_{A, V_{\mathcal{P}}}^{2}=\int_{\mathbb{R}^{N}}\left(\left|\nabla_{A} u\right|^{2}+V_{\mathcal{P}}|u|^{2}\right) \mathrm{d} x .
$$

We observe that the energy functional $J_{A, V_{\mathcal{P}}}$ on $H_{A, V_{\mathcal{P}}}^{1}\left(\mathbb{R}^{N}, \mathbb{C}\right)$ associated with (10) is given by

$$
J_{A, V_{\mathcal{P}}}(u):=\frac{1}{2}\|u\|_{A, V_{\mathcal{P}}}^{2}-\frac{1}{2 \cdot 2_{\alpha}^{*}} D(u)-\frac{\lambda}{2 p} B(u),
$$

where

and

$$
B(u)=\int_{\mathbb{R}^{N}}\left(\frac{1}{|x|^{\alpha}} *|u|^{p}\right)|u|^{p} \mathrm{~d} x=\int_{\mathbb{R}^{N}} \int_{\mathbb{R}^{N}} \frac{\left|u(x)^{p}\right||u(y)|^{p}}{|x-y|^{\alpha}} \mathrm{d} x \mathrm{~d} y
$$

$$
D(u)=\int_{\mathbb{R}^{N}}\left(\frac{1}{|x|^{\alpha}} *|u|^{2_{\alpha}^{*}}\right)|u|^{2_{\alpha}^{*}} \mathrm{~d} x=\int_{\mathbb{R}^{N}} \int_{\mathbb{R}^{N}} \frac{\left|u(x)^{2_{\alpha}^{*}}\right||u(y)|^{2_{\alpha}^{*}}}{|x-y|^{\alpha}} \mathrm{d} x \mathrm{~d} y .
$$

Remark 3.1. Notice that, by the Hardy-Littlewood-Sobolev inequality, the integral

$$
\int_{\mathbb{R}^{N}} \int_{\mathbb{R}^{N}} \frac{\left|u(x)^{s}\right||u(y)|^{s}}{|x-y|^{\alpha}} \mathrm{d} x \mathrm{~d} y
$$

is well defined if

$$
\frac{2 N-\alpha}{N} \leq s \leq \frac{2 N-\alpha}{N-2}
$$

Here, as also in [6], $\frac{2 N-\alpha}{N}$ is called the lower critical exponent and $2_{\alpha}^{*}=\frac{2 N-\alpha}{N-2}$ the upper critical exponent. This lead us to say that (11) is a critical nonlocal elliptic equation.

The Hardy-Littlewood-Sobolev inequality implies that

$$
|B(u)| \leq C_{1}(N, \alpha)\|u\|_{p}^{2 p}
$$

and

$$
|D(u)| \leq C_{2}(N, \alpha)\|u\|_{2_{\alpha}^{*}}^{2 \cdot 2_{\alpha}^{*}}
$$

for constants $C_{1}(N, \alpha)$ and $C_{2}(N, \alpha)$. For any $u \in H_{A, V_{\mathcal{P}}}^{1}\left(\mathbb{R}^{N}, \mathbb{C}\right)$ the immersions (77) imply that $B$ and $D$ are well-defined. Consequently, $J_{A, V_{\mathcal{P}}}$ is well-defined.

Observe that

$$
S_{A}=\inf _{u \in D_{A}^{1,2}\left(\mathbb{R}^{N}\right) \backslash\{0\}} \frac{\int_{\mathbb{R}^{N}}\left|\nabla_{A} u\right|^{2} \mathrm{~d} x}{D(u)^{\frac{N-2}{2 N-\alpha}}} .
$$

Definition 3.1. A function $u \in H_{A, V_{\mathcal{P}}}^{1}\left(\mathbb{R}^{N}, \mathbb{C}\right)$ is a weak solution of (10) if

$$
\left.\left.\langle u, \psi\rangle_{A, V_{\mathcal{P}}}-\mathfrak{R e} \int_{\mathbb{R}^{N}}\left(\frac{1}{|x|^{\alpha}} *|u|^{2_{\alpha}^{*}}\right)\right)|u|^{2_{\alpha}^{*}-2} u \bar{\psi} \mathrm{d} x-\lambda \mathfrak{R e} \int_{\mathbb{R}^{N}}\left(\frac{1}{|x|^{\alpha}} *|u|^{p}\right)\right)|u|^{p-2} u \bar{\psi} \mathrm{d} x=0
$$

for all $\psi \in H_{A, V_{\mathcal{P}}}^{1}\left(\mathbb{R}^{N}, \mathbb{C}\right)$. 
Since the derivative of the energy functional $J_{A, V_{\mathcal{P}}}$ is given by

$$
\left.\left.J_{A, V_{\mathcal{P}}}^{\prime}(u) \cdot \psi=\langle u, \psi\rangle_{A, V_{\mathcal{P}}}-\mathfrak{R e} \int_{\mathbb{R}^{N}}\left(\frac{1}{|x|^{\alpha}} *|u|^{2_{\alpha}^{*}}\right)\right)|u|^{2_{\alpha}^{*}-2} u \bar{\psi} \mathrm{d} x-\lambda \mathfrak{R e} \int_{\mathbb{R}^{N}}\left(\frac{1}{|x|^{\alpha}} *|u|^{p}\right)\right)|u|^{p-2} u \bar{\psi} \mathrm{d} x,
$$

we see that critical points of $J_{A, V_{\mathcal{P}}}$ are weak solutions of (10).

Note that, if $\psi=u$ we obtain

$$
J_{A, V_{\mathcal{P}}}^{\prime}(u) \cdot u:=\|u\|_{A, V_{\mathcal{P}}}^{2}-D(u)-\lambda B(u) .
$$

Lemma 7. The functional $J_{A, V_{\mathcal{P}}}$ satisfies the mountain pass geometry. Precisely,

(i) there exist $\rho, \delta>0$ such that $\left.J_{A, V_{\mathcal{P}}}\right|_{S} \geq \delta>0$ for any $u \in \mathcal{S}$, where

$$
\mathcal{S}=\left\{u \in H_{A, V_{\mathcal{P}}}^{1}\left(\mathbb{R}^{N}, \mathbb{C}\right):\|u\|_{A, V_{\mathcal{P}}}=\rho\right\}
$$

(ii) for any $u_{0} \in H_{A, V_{\mathcal{P}}}^{1}\left(\mathbb{R}^{N}, \mathbb{C}\right) \backslash\{0\}$ there exists $\tau \in(0, \infty)$ such that $\left\|\tau u_{0}\right\|_{V_{\mathcal{P}}}>\rho$ and $J_{A, V_{\mathcal{P}}}\left(\tau u_{0}\right)<0$.

Proof. Inequalities (11) and (12) yields

$$
J_{A, V_{\mathcal{P}}}(u) \geq \frac{1}{2}\|u\|_{A, V_{\mathcal{P}}}^{2}-\frac{C_{2}(\alpha, N)}{2 \cdot 2_{\alpha}^{*}}\|u\|_{A, V_{\mathcal{P}}}^{2 \cdot 2^{*}}-\frac{\lambda C_{1}(\alpha, N)}{2 p}\|u\|_{A, V_{\mathcal{P}}}^{2 p},
$$

thus implying $(i)$ if we take $\|u\|_{A, V_{\mathcal{P}}}=\rho>0$ sufficiently small.

In order to prove $(i i)$, fix $u_{0} \in H_{A, V_{\mathcal{P}}}^{1}\left(\mathbb{R}^{N}, \mathbb{C}\right) \backslash\{0\}$ and consider the function $g_{u_{0}}:(0, \infty) \rightarrow \mathbb{R}$ given by

We have

$$
g_{u_{0}}(t):=J_{A, V_{\mathcal{P}}}\left(t u_{0}\right)=\frac{1}{2}\left\|t u_{0}\right\|_{A, V_{\mathcal{P}}}^{2}-\frac{1}{2 \cdot 2_{\alpha}^{*}} D\left(t u_{0}\right)-\frac{\lambda}{2 p} B\left(t u_{0}\right) .
$$

$$
B\left(t u_{0}\right)=t^{2 p} \int_{\mathbb{R}^{N}} \int_{\mathbb{R}^{N}} \frac{\left|u_{0}(x)^{p}\right|\left|u_{0}(y)\right|^{p}}{|x-y|^{\alpha}} \mathrm{d} x \mathrm{~d} y=t^{2 p} B\left(u_{0}\right)
$$

and

$$
D\left(t u_{0}\right)=t^{2 \cdot 2_{\alpha}^{*}} \int_{\mathbb{R}^{N}} \int_{\mathbb{R}^{N}} \frac{\left|u_{0}(x)\right|^{2}\left|u_{0}(y)\right|^{2_{\alpha}^{*}}}{|x-y|^{\alpha}} \mathrm{d} x \mathrm{~d} y=t^{2 \cdot 2_{\alpha}^{*}} D\left(u_{0}\right)
$$

Thus,

$$
\begin{aligned}
g_{u_{0}}(t) & =\frac{1}{2} t^{2}\left\|u_{0}\right\|_{A, V_{\mathcal{P}}}^{2}-\frac{1}{2 \cdot 2_{\alpha}^{*}} t^{2 \cdot 2_{\alpha}^{*}} D\left(u_{0}\right)-\frac{\lambda}{2 p} t^{2 p} B\left(u_{0}\right) \\
& =\frac{1}{2} t^{2 \cdot 2_{\alpha}^{*}}\left(\frac{\left\|u_{0}\right\|_{A, V_{\mathcal{P}}}^{2}}{t^{\left(2\left(2_{\alpha}^{*}-1\right)\right)}}-\frac{1}{2_{\alpha}^{*}} D\left(u_{0}\right)-\frac{\lambda}{p} \frac{B\left(u_{0}\right)}{t^{\left(2\left(2_{\alpha}^{*}-p\right)\right)}}\right)
\end{aligned}
$$

Since $1<p<2_{\alpha}^{*}$, we have

$$
\lim _{t \rightarrow+\infty} J_{A, V_{\mathcal{P}}}\left(t u_{0}\right)=-\infty
$$

completing the proof of $(i i)$.

The mountain pass theorem without the PS condition (see [31, Theorem 1.15]) yields a Palais-Smale sequence $\left(u_{n}\right) \subset H_{A, V_{\mathcal{P}}}^{1}\left(\mathbb{R}^{N}, \mathbb{C}\right)$ such that

$$
J_{A, V_{\mathcal{P}}}^{\prime}\left(u_{n}\right) \rightarrow 0 \quad \text { and } \quad J_{A, V_{\mathcal{P}}}\left(u_{n}\right) \rightarrow c_{\lambda},
$$

where

$$
c_{\lambda}=\inf _{\alpha \in \Gamma \in[0,1]} \max _{t \in V_{\mathcal{P}}}(\gamma(t)),
$$

and $\Gamma=\left\{\gamma \in C^{1}\left([0,1], H_{A, V_{\mathcal{P}}}^{1}\left(\mathbb{R}^{N}, \mathbb{C}\right)\right): \gamma(0)=0, J_{A, V_{\mathcal{P}}}(\gamma(1))<0\right\}$.

Lemma 8. Suppose that $u_{n} \rightarrow u_{0}$ in $H_{A, V_{\mathcal{P}}}^{1}\left(\mathbb{R}^{N}, \mathbb{C}\right)$. Then

$$
\frac{1}{|x|^{\alpha}} *\left|u_{n}\right|^{s} \rightarrow \frac{1}{|x|^{\alpha}} *\left|u_{0}\right|^{s} \text { in } L^{\frac{2 N}{\alpha}}\left(\mathbb{R}^{N}\right)
$$

for all $\frac{2 N-\alpha}{N} \leq s \leq 2_{\alpha}^{*}$. 
Proof. In this proof we adapt some ideas of [7]. We can suppose that $\left|u_{n}(x)\right|^{s} \rightarrow\left|u_{0}(x)\right|^{s}$ a.e. in $\mathbb{R}^{N}$ and, as consequence of the immersion (77), $\left|u_{n}\right|^{s}$ is bounded in $L^{\frac{2 N}{2 N-\alpha}}\left(\mathbb{R}^{N}\right)$. Thus, Lemma 6 allows us to conclude that

$$
\left|u_{n}(x)\right|^{s} \rightarrow\left|u_{0}(x)\right|^{s} \text { in } L^{\frac{2 N}{2 N-\alpha}}\left(\mathbb{R}^{N}, \mathbb{C}\right) .
$$

The Hardy-Littlewood-Sobolev inequality allows us to conclude that

$$
\frac{1}{|x|^{\alpha}} * w(x) \in L^{\frac{2 N}{\alpha}}\left(\mathbb{R}^{N}\right)
$$

for all $w \in L^{\frac{2 N}{2 N-\alpha}}\left(\mathbb{R}^{N}\right)$. So, we have a continuous linear map from $L^{\frac{2 N}{2 N-\alpha}}\left(\mathbb{R}^{N}\right)$ to $L^{\frac{2 N}{\alpha}}\left(\mathbb{R}^{N}\right)$. A new application of Lemma 6 yields (15).

Corollary 9. Suppose that $u_{n} \rightarrow u_{0}$ and consider

$$
\left.B^{\prime}\left(u_{n}\right) \cdot \psi=\mathfrak{R e} \int_{\mathbb{R}^{N}}\left(\frac{1}{|x|^{\alpha}} *\left|u_{n}\right|^{p}\right)\right)\left|u_{n}\right|^{p-2} u_{n} \bar{\psi} \mathrm{d} x
$$

and

$$
\left.D^{\prime}\left(u_{n}\right) \cdot \psi=\mathfrak{R e} \int_{\mathbb{R}^{N}}\left(\frac{1}{|x|^{\alpha}} *\left|u_{n}\right|^{2_{\alpha}^{*}}\right)\right)\left|u_{n}\right|^{2_{\alpha}^{*}-2} u_{n} \bar{\psi} \mathrm{d} x,
$$

for $\psi \in C_{c}^{\infty}\left(\mathbb{R}^{N}, \mathbb{C}\right)$. Then $B^{\prime}\left(u_{n}\right) \cdot \psi \rightarrow B^{\prime}\left(u_{0}\right) \cdot \psi$ and $D^{\prime}\left(u_{n}\right) \cdot \psi \rightarrow D^{\prime}\left(u_{0}\right) \cdot \psi$.

Proof. The immersion (7) guarantees that $\left|u_{n}\right|^{p-2} u_{n}$ is bounded in $L^{\frac{2 N}{N+2-\alpha}}\left(\mathbb{R}^{N}, \mathbb{C}\right)$. Since we can suppose that $\left|u_{n}(x)\right|^{p} \rightarrow\left|u_{0}(x)\right|^{p}$ a.e. in $\mathbb{R}^{N}$, by applying Lemma 6, we conclude that

$$
\left|u_{n}\right|^{p-2} u_{n} \rightarrow\left|u_{0}\right|^{p-2} u \quad \text { in } L^{\frac{2 N}{N+2-\alpha}}\left(\mathbb{R}^{N}, \mathbb{C}\right)
$$

for all $\frac{2 N-\alpha}{N} \leq p \leq 2_{\alpha}^{*}$, as $n \rightarrow+\infty$.

Combining (15) with (16) yields

$$
\left(\frac{1}{|x|^{\alpha}} *\left|u_{n}\right|^{p}\right)\left|u_{n}\right|^{p-2} u_{n} \rightarrow\left(\frac{1}{|x|^{\alpha}} *\left|u_{0}\right|^{p}\right)\left|u_{0}\right|^{p-2} u_{0} \text { in } L^{\frac{2 N}{N+2}}\left(\mathbb{R}^{N}\right)
$$

as $n \rightarrow+\infty$, for all $\frac{2 N-\alpha}{N} \leq p \leq 2_{\alpha}^{*}$. Consequently, for $\psi \in C_{c}^{\infty}\left(\mathbb{R}^{N}, \mathbb{C}\right)$, it follows that

$$
\mathfrak{R e} \int_{\mathbb{R}^{N}}\left(\frac{1}{|x|^{\alpha}} *\left|u_{n}\right|^{p}\right)\left|u_{n}\right|^{p-2} u_{n} \bar{\psi} \mathrm{d} x \rightarrow \mathfrak{R e} \int_{\mathbb{R}^{N}}\left(\frac{1}{|x|^{\alpha}} *\left|u_{0}\right|^{p}\right)\left|u_{0}\right|^{p-2} u_{0} \bar{\psi} \mathrm{d} x
$$

and

that is,

$$
\mathfrak{R e} \int_{\mathbb{R}^{N}}\left(\frac{1}{|x|^{\alpha}} *\left|u_{n}\right|^{2_{\alpha}^{*}}\right)\left|u_{n}\right|^{2_{\alpha}^{*}-2} u_{n} \bar{\psi} \mathrm{d} x \rightarrow \mathfrak{R e} \int_{\mathbb{R}^{N}}\left(\frac{1}{|x|^{\alpha}} *\left|u_{0}\right|^{2_{\alpha}^{*}}\right)\left|u_{0}\right|^{2_{\alpha}^{*}-2} u_{0} \bar{\psi} \mathrm{d} x,
$$

$$
B^{\prime}\left(u_{n}\right) \cdot \psi \rightarrow B^{\prime}\left(u_{0}\right) \cdot \psi \quad \text { and } \quad D^{\prime}\left(u_{n}\right) \cdot \psi \rightarrow D^{\prime}\left(u_{0}\right) \cdot \psi
$$

Lemma 10. If $\left(u_{n}\right) \subset H_{A, V_{\mathcal{P}}}^{1}\left(\mathbb{R}^{N}, \mathbb{C}\right)$ is a $(P S)_{\lambda}$ sequence for $J_{A, V_{\mathcal{P}}}$, then $\left(u_{n}\right)$ is bounded. In addition, if $u_{n} \rightarrow u$ weakly in $H_{A, V_{\mathcal{P}}}^{1}\left(\mathbb{R}^{N}, \mathbb{C}\right)$ as $n \rightarrow \infty$, then $u$ is ground state solution for problem (10).

Proof. Standard arguments prove that $\left(u_{n}\right)$ is bounded in $H_{A, V_{\mathcal{P}}}^{1}\left(\mathbb{R}^{N}, \mathbb{C}\right)$. Then, up to a subsequence, we have $u_{n} \rightarrow u$ weakly in $H_{A, V_{\mathcal{P}}}^{1}\left(\mathbb{R}^{N}, \mathbb{C}\right)$ as $n \rightarrow \infty$.

From Corollary 9 it follows that, for all $\psi \in H_{A, V_{\mathcal{P}}}^{1}\left(\mathbb{R}^{N}, \mathbb{C}\right)$, we have

$$
\mathfrak{R e} \int_{\mathbb{R}^{N}}\left(\frac{1}{|x|^{\alpha}} *\left|u_{n}\right|^{p}\right)|u|^{p-2} u_{n} \bar{\psi} \mathrm{d} x=\mathfrak{R e} \int_{\mathbb{R}^{N}}\left(\frac{1}{|x|^{\alpha}} *|u|^{p}\right)|u|^{p-2} u \bar{\psi} \mathrm{d} x+o_{n}(1), \text { as } n \rightarrow \infty,
$$

where $s=p$ or $s=2_{\alpha}^{*}$.

Thus, since for all $\psi \in C_{c}^{\infty}\left(\mathbb{R}^{N}, \mathbb{C}\right)$ we have $J_{A, V_{\mathcal{P}}}^{\prime}\left(u_{n}\right) \cdot \psi=o_{n}(1)$, we obtain

$$
J_{A, V}^{\prime}(u) \cdot \psi=0, \quad \forall \psi \in H_{A, V_{\mathcal{P}}}^{1}\left(\mathbb{R}^{N}, \mathbb{C}\right),
$$

that is, $u$ is a ground state solution for (10). 
We now consider the Nehari manifold associated with the $J_{A, V_{\mathcal{P}}}$.

$$
\mathcal{M}_{A, V_{\mathcal{P}}}=\left\{u \in H_{A, V_{\mathcal{P}}}^{1}\left(\mathbb{R}^{N}, \mathbb{C}\right) \backslash\{0\}:\|u\|_{A, V_{\mathcal{P}}}^{2}=D(u)+\lambda B(u)\right\} .
$$

Lemma 11. There exists a unique $t_{u}=t_{u}(u)>0$ such that $t_{u} u \in \mathcal{M}_{A, V_{\mathcal{P}}}$ for all $u \in H_{A, V_{\mathcal{P}}}^{1}\left(\mathbb{R}^{N}, \mathbb{C}\right) \backslash\{0\}$ and $J_{A, V_{\mathcal{P}}}\left(t_{u} u\right)=\max _{t \geq 0} J_{A, V_{\mathcal{P}}}(t u)$. Moreover $c_{\lambda}=c_{\lambda}^{*}=c_{\lambda}^{* *}$, where

$$
c_{\lambda}^{*}=\inf _{u \in \mathcal{M}_{A, V}} J_{A, V_{\mathcal{P}}}(u) \quad \text { and } \quad c_{\lambda}^{* *}=\inf _{u \in H_{A, V_{\mathcal{P}}}^{1}\left(\mathbb{R}^{N}, \mathbb{C}\right) \backslash\{0\}} \max _{t \geq 0} J_{A, V_{\mathcal{P}}}(t u) .
$$

Proof. Let $u \in H_{A, V_{\mathcal{P}}}^{1}\left(\mathbb{R}^{N}, \mathbb{C}\right) \backslash\{0\}$ and $g_{u}$ defined on $(0,+\infty)$ given by

$$
g_{u}(t)=J_{A, V_{\mathcal{P}}}(t u) .
$$

By the mountain pass geometry (Lemma 7), there exists $t_{u}>0$ such that

$$
g_{u}\left(t_{u}\right)=\max _{t \geq 0} g_{u}(t)=\max _{t \geq 0} J_{A, V_{\mathcal{P}}}\left(t_{u} u\right) .
$$

Hence

$$
0=g_{u}^{\prime}\left(t_{u}\right)=J_{A, V_{\mathcal{P}}}^{\prime}\left(t_{u} u\right) \cdot u=J_{A, V_{\mathcal{P}}}^{\prime}\left(t_{u} u\right) \cdot t_{u} u,
$$

implying that $t_{u} u \in \mathcal{M}_{A, V_{\mathcal{P}}}$, as consequence of (14). We now show that $t_{u}$ is unique. To this end, we suppose that there exists $s_{u}>0$ such that $s_{u} u \in \mathcal{M}_{A, V_{\mathcal{P}}}$. Thus, we have both

$$
\|u\|_{A, V_{\mathcal{P}}}^{2}=t_{u}^{2\left(2_{\alpha}^{*}-1\right)} D(u)+\lambda t_{u}^{2(p-1)} B(u) \quad \text { and } \quad\|u\|_{A, V_{\mathcal{P}}}^{2}=s_{u}^{2\left(2_{\alpha}^{*}-1\right)} D(u)+\lambda s_{u}^{2(p-1)} B(u) .
$$

Hence

$$
0=\left(t_{u}^{2^{\left(22_{\alpha}^{*}-1\right)}}-s_{u}^{2\left(2_{\alpha}^{*}-1\right)}\right) D(u)+\lambda\left(t_{u}^{2(p-1)}-s_{u}^{2(p-1)}\right) B(u) .
$$

Since both terms in parentheses have the same sign if $t_{u} \neq s_{u}$ and we also have $B(u)>0, D(u)>0$ and $\lambda>0$, it follows that $t_{u}=s_{u}$.

Now, the rest of the proof follows arguments similar to that found in [1, 19, 29, 31.

The following result controls the level $c_{\lambda}$ of a Palais-Smale sequence of $J_{A, V_{\mathcal{P}}}$.

Lemma 12. Let $\left(u_{n}\right) \subset H_{A, V_{\mathcal{P}}}^{1}\left(\mathbb{R}^{N}, \mathbb{C}\right)$ a $(P S)_{c_{\lambda}}$ sequence for $J_{A, V_{\mathcal{P}}}$ such that

$$
u_{n} \rightarrow 0 \text { weakly in } H_{A, V_{\mathcal{P}}}^{1}\left(\mathbb{R}^{N}, \mathbb{C}\right) \text {, as } n \rightarrow \infty \text {, }
$$

with

$$
c_{\lambda}<\frac{N+2-\alpha}{2(2 N-\alpha)} S_{A}^{\frac{2 N-\alpha}{N-\alpha+2}} .
$$

Then the sequence $\left(u_{n}\right)$ verifies either

(i) $u_{n} \rightarrow 0$ strongly in $H_{A, V_{\mathcal{P}}}^{1}\left(\mathbb{R}^{N}, \mathbb{C}\right)$, as $n \rightarrow \infty$,

or

(ii) There exists a sequence $\left(y_{n}\right) \subset \mathbb{R}^{N}$ and constants $r, \theta>0$ such that

$$
\limsup _{n \rightarrow \infty} \int_{B_{r}\left(y_{n}\right)}\left|u_{n}\right|^{2} \mathrm{~d} x \geq \theta
$$

where $B_{r}(y)$ denotes the ball in $\mathbb{R}^{N}$ of center at $y$ and radius $r>0$.

Proof. Suppose that (ii) does not hold. Applying a result by Lions [31, Lemma 1.21], it follows from inequality (11) that

$$
B\left(u_{n}\right) \rightarrow 0, \quad \text { as } \quad n \rightarrow \infty .
$$

Since $J_{A, V_{\mathcal{P}}}^{\prime}\left(u_{n}\right) u_{n}=o_{n}(1)$ as $n \rightarrow \infty$, we obtain

$$
\left\|u_{n}\right\|_{A, V_{\mathcal{P}}}^{2}=D\left(u_{n}\right)+o_{n}(1) \text { as } n \rightarrow \infty .
$$

Let us suppose that

$$
\left\|u_{n}\right\|_{A, V_{\mathcal{P}}}^{2} \rightarrow \ell(\ell>0) \quad \text { as } n \rightarrow \infty .
$$


Thus, as consequence of (18), we have

$$
D\left(u_{n}\right) \rightarrow \ell, \quad \text { as } \quad n \rightarrow \infty
$$

Since

making $n \rightarrow \infty$ yields

$$
J_{A, V}\left(u_{n}\right)=\frac{1}{2}\|u\|_{A, V}^{2}-\frac{\lambda}{2 p} B\left(u_{n}\right)-\frac{1}{2 \cdot 2_{\alpha}^{*}} D\left(u_{n}\right),
$$

$$
c_{\lambda}=\frac{\ell}{2}\left(1-\frac{1}{2_{\alpha}^{*}}\right)=\ell\left(\frac{N+2-\alpha}{2(2 N-\alpha)}\right) .
$$

On the other hand, it follows from (13) that

$$
\left\|u_{n}\right\|_{A, V_{\mathcal{P}}}^{2} \geq \int_{\mathbb{R}^{N}}\left|\nabla_{A} u_{n}\right|^{2} \mathrm{~d} x \geq S_{A}\left(D\left(u_{n}\right)\right)^{\frac{N-2}{2 N-\alpha}}, \quad \forall u \in D_{A}^{1,2}\left(\mathbb{R}^{N}\right) .
$$

Thus,

$$
\ell \geq\left(S_{A}\right)^{\frac{2 N-\alpha}{N+2-\alpha}}
$$

and from (19) and (20) we conclude that $c_{\lambda} \geq \frac{N+2-\alpha}{2(2 N-\alpha)} S_{A}^{\frac{2 N-\alpha}{N+2-\alpha}}$, which is a contradiction. Therefore, $(i)$ is valid and the proof is complete.

We now state our result about the periodic problem (10).

Theorem 13. Under the hypotheses already stated on $A$ and $\alpha$, suppose that $\left(V_{1}\right)$ is valid. Then problem (10) has at least one ground state solution if either

(i) $\frac{N+2-\alpha}{N-2}<p<2_{\alpha}^{*}, N=3,4$ and $\lambda>0$;

(ii) $\frac{2 N-\alpha}{N}<p \leq \frac{N+2-\alpha}{N-2}, N=3,4$ and $\lambda$ sufficiently large;

(iii) $\frac{2 N-\alpha-2}{N-2}<p<2_{\alpha}^{*}, N \geq 5$ and $\lambda>0$;

(iv) $\frac{2 N-\alpha}{N}<p \leq \frac{2 N-\alpha-2}{N-2}, N \geq 5$ and $\lambda$ sufficiently large.

Proof. Let $c_{\lambda}$ be the mountain pass level and consider a sequence $\left(u_{n}\right) \subset H_{A, V_{\mathcal{P}}}^{1}\left(\mathbb{R}^{N}, \mathbb{C}\right)$ such that

$$
J_{A, V_{\mathcal{P}}}^{\prime}\left(u_{n}\right) \rightarrow 0 \quad \text { and } \quad J_{A, V_{\mathcal{P}}}\left(u_{n}\right) \rightarrow c_{\lambda} .
$$

Claim. We affirm that $c_{\lambda}<\frac{N+2-\alpha}{2(2 N-\alpha)}\left(S_{A}\right)^{\frac{2 N-\alpha}{N+2-\alpha}}$, a result that will be shown after completing our proof, since it is very technical.

Lemma 10 guarantees that $\left(u_{n}\right)$ is bounded. So, passing to a subsequence if necessary, there is $u \in H_{A, V_{\mathcal{P}}}^{1}\left(\mathbb{R}^{N}, \mathbb{C}\right)$ such that

$$
u_{n} \rightarrow u \text { in } H_{A, V_{\mathcal{P}}}^{1}\left(\mathbb{R}^{N}, \mathbb{C}\right), \quad u_{n} \rightarrow u \text { in } L_{l o c}^{2}\left(\mathbb{R}^{N}, \mathbb{C}\right) \quad \text { and } \quad u_{n} \rightarrow u \text { a.e. } x \in \mathbb{R}^{N} .
$$

If $u \neq 0$ we are done. If $u=0$, it follows from Lemma 12 the existence of $\theta>0$ and $\left(y_{n}\right) \subset \mathbb{R}^{N}$ such that

$$
\limsup _{n \rightarrow \infty} \int_{B_{r}\left(y_{n}\right)}\left|u_{n}\right|^{2} \mathrm{~d} x \geq \theta .
$$

A direct computation shows that we can assume that $\left(y_{n}\right) \subset \mathbb{Z}^{N}$. Let

$$
v_{n}(x):=u_{n}\left(x+y_{n}\right) .
$$

Since both $V_{\mathcal{P}}$ and $A$ are $\mathbb{Z}^{N}$-periodic, we have

$$
\left\|v_{n}\right\|_{A, V_{\mathcal{P}}}=\left\|u_{n}\right\|_{A, V_{\mathcal{P}}} \quad J_{A, V_{\mathcal{P}}}\left(v_{n}\right)=J_{A, V_{\mathcal{P}}}\left(u_{n}\right) \quad \text { and } \quad J_{A, V_{\mathcal{P}}}^{\prime}\left(v_{n}\right) \rightarrow 0, \quad \text { as } n \rightarrow \infty .
$$

Therefore there exists $v \in H_{A, V_{\mathcal{P}}}^{1}$ such that $v_{n} \rightarrow v$ weakly in $H_{A, V_{\mathcal{P}}}^{1}\left(\mathbb{R}^{N}, \mathbb{C}\right)$ and $v_{n} \rightarrow v$ in $L_{l o c}^{2}\left(\mathbb{R}^{N}, \mathbb{C}\right)$.

We claim that $v \neq 0$. In fact, it follows from (21)

$$
0<\theta \leq\left\|v_{n}\right\|_{L^{2}\left(B_{r}(0)\right)} \leq\left\|v_{n}-v\right\|_{L^{2}\left(B_{r}(0)\right)}+\|v\|_{L^{2}\left(B_{r}(0)\right)} .
$$

Since $v_{n} \rightarrow v$ in $L_{l o c}^{2}\left(\mathbb{R}^{N}\right)$, we have $\left\|v_{n}-v\right\|_{L^{2}\left(B_{r}(0)\right)} \rightarrow 0$ as $n \rightarrow \infty$, proving our claim.

But Corollary9 guarantees that $J_{A, V_{\mathcal{P}}}^{\prime}\left(v_{n}\right) \cdot \psi \rightarrow J_{A, V_{\mathcal{P}}}^{\prime}\left(v_{n}\right) \cdot \psi$ and it follows that $J_{A, V_{\mathcal{P}}}^{\prime}(v) \cdot \psi=0$. Consequently, $v$ is a ground state solution of problem (10). 
We now prove the postponed Claim, that is, we show that $c_{\lambda}<\frac{N+2-\alpha}{2(2 N-\alpha)}\left(S_{A}\right)^{\frac{2 N-\alpha}{N+2-\alpha}}$. Observe that, once proved the existence of $u_{\epsilon}$ as in our next result, then

$$
0<c_{\lambda}=\inf _{\alpha \in \Gamma} \max _{t \in[0,1]} J_{A, V_{\mathcal{P}}}(\gamma(t)) \leq \sup _{t \geq 0} J_{A, V_{\mathcal{P}}}\left(t u_{\varepsilon}\right)<\frac{N+2-\alpha}{2(2 N-\alpha)}\left(S_{A}\right)^{\frac{2 N-\alpha}{N+2-\alpha}} .
$$

Lemma 14. There exists $u_{\varepsilon}$ such that

$$
\sup _{t \geq 0} J_{A, V_{\mathcal{P}}}\left(t u_{\varepsilon}\right)<\frac{N+2-\alpha}{2(2 N-\alpha)}\left(S_{A}\right)^{\frac{2 N-\alpha}{N+2-\alpha}} .
$$

provided that either

(i) $\frac{N+2-\alpha}{N-2}<p<2_{\alpha}^{*}, N=3,4$ and $\lambda>0$;

(ii) $\frac{2 N-\alpha}{N}<p \leq \frac{N+2-\alpha}{N-2}, N=3,4$ and $\lambda$ sufficiently large;

(iii) $\frac{2 N-2-\alpha}{N-2}<p<2_{\alpha}^{*}, N \geq 5$ and $\lambda>0$;

(iv) $\frac{2 N-\alpha}{N}<p \leq \frac{2 N-2-\alpha}{N-2}, N \geq 5$ and $\lambda$ sufficiently large.

The arguments of this proof were adapted from the articles [20, 25]. Observe that the conditions stated in this result are exactly the same of Theorem 1 and Theorem 13 .

Proof. We know that $U(x)=\frac{[N(N-2)]^{\frac{N-2}{4}}}{\left(1+|x|^{2}\right)^{\frac{N-2}{2}}}$ is a minimizer for $S$, the best Sobolev constant of the immersion $D^{1,2}\left(\mathbb{R}^{N}\right) \hookrightarrow L^{2^{*}}\left(\mathbb{R}^{N}\right)$ (see 31, Theorem 1.42] or [10, Section 3]) and also a minimizer for $S_{H, L}$, according to Proposition 4

If $B_{r}$ denotes the ball in $\mathbb{R}^{N}$ of center at origin and radius $r$, consider the balls $B_{\delta}$ and $B_{2 \delta}$ and take $\psi \in C_{0}^{\infty}\left(\mathbb{R}^{N}\right)$ such that, for a constant $C>0$,

$$
\psi(x)=\left\{\begin{array}{ll}
1, & \text { if } x \in B_{\delta}, \\
0, & \text { if } x \in \mathbb{R}^{N} \backslash B_{2 \delta},
\end{array} \quad 0 \leq|\psi(x)| \leq 1, \quad|D \psi(x)| \leq C, \quad \forall x \in \mathbb{R}^{N} .\right.
$$

We define, for $\varepsilon>0$,

$$
U_{\varepsilon}(x):=\varepsilon^{(2-N) / 2} U\left(\frac{x}{\varepsilon}\right) \quad \text { and } \quad u_{\varepsilon}(x):=\psi(x) U_{\varepsilon}(x)
$$

In the proof we apply the estimates

$$
\int_{\mathbb{R}^{N}}\left|\nabla u_{\varepsilon}\right|^{2} \mathrm{~d} x=C(N, \alpha)^{\frac{N-2}{2 N-\alpha} \cdot \frac{N}{2}} S_{A}^{\frac{N}{2}}+O\left(\varepsilon^{N-2}\right)
$$

and

$$
\int_{\mathbb{R}^{N}} \int_{\mathbb{R}^{N}} \frac{\left|u_{\varepsilon}(x)\right|^{2_{\alpha}^{*}}\left|u_{\varepsilon}(y)\right|^{2_{\alpha}^{*}}}{|x-y|^{\alpha}} \mathrm{d} x \mathrm{~d} y \geq C(N, \alpha)^{\frac{N}{2}} S_{A}^{\frac{2 N-\alpha}{2}}-O\left(\varepsilon^{N-\frac{\alpha}{2}}\right),
$$

which were obtained by Gao and Yang [21].

Case 1. $\frac{N+2-\alpha}{N-2}<p<2_{\alpha}^{*}$ and $N=3,4$ or $\frac{2 N-2-\alpha}{N-2}<p<2_{\alpha}^{*}$ and $N \geq 5$.

Proof of Case 1. Consider the function $f:[0,+\infty) \rightarrow \mathbb{R}$ defined by

$$
f(t)=J_{A, V_{\mathcal{P}}}\left(t u_{\varepsilon}\right)=\frac{t^{2}}{2}\left\|u_{\varepsilon}\right\|_{A, V_{\mathcal{P}}}^{2}-\frac{t^{2 \cdot 2_{\alpha}^{*}}}{2 \cdot 2_{\alpha}^{*}} D\left(u_{\varepsilon}\right)-\frac{\lambda t^{2 p}}{2 p} B\left(u_{\varepsilon}\right) .
$$

The mountain pass geometry (Lemma 7) implies the existence of $t_{\varepsilon}>0$ such that $\sup _{t \geq 0} J_{A, V_{\mathcal{P}}}\left(t u_{\varepsilon}\right)=J_{A, V_{\mathcal{P}}}\left(t_{\varepsilon} u_{\varepsilon}\right)$. Since $t_{\varepsilon}>0, B\left(u_{\varepsilon}\right)>0$ and $f^{\prime}\left(t_{\varepsilon}\right)=0$, we obtain

$$
0<t_{\varepsilon}<\left(\frac{\left\|u_{\varepsilon}\right\|_{A, V_{\mathcal{P}}}^{2}}{D\left(u_{\varepsilon}\right)}\right)^{\frac{1}{2\left(2_{\alpha}^{*}-1\right)}}:=S_{A}(\varepsilon),
$$


thus implying

$$
\left\|u_{\varepsilon}\right\|_{A, V_{\mathcal{P}}}^{2}=D\left(u_{\varepsilon}\right)\left(S_{A}(\varepsilon)\right)^{2\left(2_{\alpha}^{*}-1\right)} .
$$

Now define $g:\left[0, S_{A}(\varepsilon)\right] \rightarrow \mathbb{R}$ by

$$
g(t)=\frac{t^{2}}{2}\left\|u_{\varepsilon}\right\|_{A, V_{\mathcal{P}}}^{2}-\frac{t^{2 \cdot 2_{\alpha}^{*}}}{2 \cdot 2_{\alpha}^{*}} D\left(u_{\varepsilon}\right)
$$

So,

$$
g(t)=\frac{t^{2}}{2} D\left(u_{\varepsilon}\right)\left(S_{A}(\varepsilon)\right)^{2\left(2_{\alpha}^{*}-1\right)}-\frac{t^{2 \cdot 2_{\alpha}^{*}}}{2 \cdot 2_{\alpha}^{*}} D\left(u_{\varepsilon}\right) .
$$

Since $t>0$ and $D\left(u_{\varepsilon}\right)>0$, it follows that $g^{\prime}(t)>0$, and, consequently, $g$ is increasing in this interval. Thus,

$$
0<g\left(t_{\varepsilon}\right)<\frac{N+2-\alpha}{2(2 N-\alpha)} D\left(u_{\varepsilon}\right)\left(S_{A}(\varepsilon)\right)^{2 \cdot 2_{\alpha}^{*}} .
$$

We conclude that

$$
D\left(u_{\varepsilon}\right)\left(S_{A}(\varepsilon)\right)^{2 \cdot 2_{\alpha}^{*}}=\frac{\left(\left\|u_{\varepsilon}\right\|_{A, V_{\mathcal{P}}}^{2}\right)^{\frac{2 N-\alpha}{N+2-\alpha}}}{D\left(u_{\varepsilon}\right)^{\frac{N-2}{N+2-\alpha}}}
$$

and therefore

$$
0<g\left(t_{\varepsilon}\right)<\frac{N+2-\alpha}{2(2 N-\alpha)} \cdot \frac{\left(\left\|u_{\varepsilon}\right\|_{A, V_{\mathcal{P}}}^{2}\right)^{\frac{2 N-\alpha}{N+2-\alpha}}}{D\left(u_{\varepsilon}\right)^{\frac{N-2}{N+2-\alpha}}} .
$$

Since $J_{A, V_{\mathcal{P}}}\left(t u_{\varepsilon}\right)=g(t)-\frac{\lambda}{2 p} t^{2 p} B\left(u_{\varepsilon}\right)$, we have

$$
J_{A, V_{\mathcal{P}}}\left(t_{\varepsilon} u_{\varepsilon}\right)<\frac{N+2-\alpha}{2(2 N-\alpha)}\left(\frac{\left\|u_{\varepsilon}\right\|_{A, V_{\mathcal{P}}}^{2}}{D\left(u_{\varepsilon}\right)^{\frac{N-2}{2 N-\alpha}}}\right)^{\frac{2 N-\alpha}{N+2-\alpha}}-\frac{\lambda}{2 p} t_{\varepsilon}^{2 p} B\left(u_{\varepsilon}\right) .
$$

But $\left\|u_{\varepsilon}\right\|_{A, V_{\mathcal{P}}}^{2}=\int_{\mathbb{R}^{N}}\left|\nabla u_{\varepsilon}\right|^{2} \mathrm{~d} x+\int_{\mathbb{R}^{N}}\left(|A(x)|^{2}+V_{\mathcal{P}}(x)\left|u_{\varepsilon}\right|^{2}\right) \mathrm{d} x$ implies

$$
\frac{\left\|u_{\varepsilon}\right\|_{A, V_{\mathcal{P}}}^{2}}{D\left(u_{\varepsilon}\right)^{\frac{N-2}{2 N-\alpha}}}=\frac{1}{\left(D\left(u_{\varepsilon}\right)\right)^{\frac{N-2}{2 N-\alpha}}} \int_{\mathbb{R}^{N}}\left|\nabla u_{\varepsilon}\right|^{2} \mathrm{~d} x+\frac{1}{\left(D\left(u_{\varepsilon}\right)\right)^{\frac{N-2}{2 N-\alpha}}} \int_{\mathbb{R}^{N}}\left(|A(x)|^{2}+V_{\mathcal{P}}(x)\left|u_{\varepsilon}\right|^{2}\right) \mathrm{d} x .
$$

Therefore, we conclude that

$$
\begin{aligned}
J_{A, V_{\mathcal{P}}}\left(t_{\varepsilon} u_{\varepsilon}\right)< & \frac{N+2-\alpha}{2(2 N-\alpha)}\left(\frac{1}{\left(D\left(u_{\varepsilon}\right)\right)^{\frac{N-2}{2 N-\alpha}}} \int_{\mathbb{R}^{N}}\left|\nabla u_{\varepsilon}\right|^{2} \mathrm{~d} x\right. \\
& \left.+\frac{1}{\left(D\left(u_{\varepsilon}\right)\right)^{\frac{N-2}{2 N-\alpha}}} \int_{\mathbb{R}^{N}}\left(|A(x)|^{2}+V_{\mathcal{P}}(x)\right)\left|u_{\varepsilon}\right|^{2} \mathrm{~d} x\right)^{\frac{2 N-\alpha}{N+2-\alpha}}-\frac{\lambda}{2 p} t_{\varepsilon}^{2 p} B\left(u_{\varepsilon}\right) .
\end{aligned}
$$

Since, for all $\beta \geq 1$ and any $a, b>0$ we have $(a+b)^{\beta} \leq a^{\beta}+\beta(a+b)^{\beta-1} b$, considering

$$
a=\frac{1}{\left(D\left(u_{\varepsilon}\right)\right)^{\frac{N-2}{2 N-\alpha}}} \int_{\mathbb{R}^{N}}\left|\nabla u_{\varepsilon}\right|^{2} \mathrm{~d} x, \quad b=\frac{1}{\left(D\left(u_{\varepsilon}\right)\right)^{\frac{N-2}{2 N-\alpha}}} \int_{\mathbb{R}^{N}}\left(|A(x)|^{2}+V_{\mathcal{P}}(x)\left|u_{\varepsilon}\right|^{2}\right) \mathrm{d} x \quad \text { and } \quad \beta=\frac{2 N-\alpha}{N+2-\alpha},
$$


it follows

$$
\begin{aligned}
J_{A, V_{\mathcal{P}}}\left(t_{\varepsilon} u_{\varepsilon}\right)< & \frac{N+2-\alpha}{2(2 N-\alpha)}\left[\left(\frac{1}{\left(D\left(u_{\varepsilon}\right)\right)^{\frac{N-2}{2 N-\alpha}}} \int_{\mathbb{R}^{N}}\left|\nabla u_{\varepsilon}\right|^{2} \mathrm{~d} x\right)^{\frac{2 N-\alpha}{N+2-\alpha}}\right. \\
& +\frac{2 N-\alpha}{N+2-\alpha}\left(\frac{1}{D\left(u_{\varepsilon}\right)^{\frac{N-2}{2 N-\alpha}}} \int_{\mathbb{R}^{N}}\left|\nabla u_{\varepsilon}\right|^{2} \mathrm{~d} x+\frac{1}{\left(D\left(u_{\varepsilon}\right)^{\frac{N-2}{2 N-\alpha}}\right.} \int_{\mathbb{R}^{N}}\left(|A(x)|^{2}+V_{\mathcal{P}}(x)\left|u_{\varepsilon}\right|^{2}\right) \mathrm{d} x\right)^{\frac{N-2}{N+2-\alpha}} \\
& \left.\cdot \frac{1}{\left(\left(D\left(u_{\varepsilon}\right)\right)^{\frac{N-2}{2 N-\alpha}}\right.} \int_{\mathbb{R}^{N}}\left(|A(x)|^{2}+V_{\mathcal{P}}(x)\left|u_{\varepsilon}\right|^{2}\right) \mathrm{d} x\right]-\frac{\lambda}{2 p} t_{\varepsilon}^{2 p} B\left(u_{\varepsilon}\right) .
\end{aligned}
$$

Taking into account (24) and (25), we conclude that

$$
\left(\frac{1}{\left(D\left(u_{\varepsilon}\right)\right)^{\frac{N-2}{2 N-\alpha}}} \int_{\mathbb{R}^{N}}\left|\nabla u_{\varepsilon}\right|^{2} \mathrm{~d} x\right)^{\frac{2 N-\alpha}{N+2-\alpha}} \leq\left(\frac{(C(N, \alpha))^{\frac{N-2}{2 N-\alpha} \cdot \frac{N}{2}} \cdot S_{H, L}^{\frac{N}{2}}+O\left(\varepsilon^{N-2}\right)}{\left(C(N, \alpha)^{\frac{N}{2}} S_{H, L}^{\frac{2 N-\alpha}{2}}-O\left(\varepsilon^{\frac{2 N-\alpha}{2}}\right)\right)^{\frac{N-2}{2 N-\alpha}}}\right)^{\frac{2 N-\alpha}{N+2-\alpha}}
$$

We also have

$$
\left(\frac{(C(N, \alpha))^{\frac{N-2}{2 N-\alpha} \cdot \frac{N}{2}}\left(S_{H, L}\right)^{\frac{N}{2}}+O\left(\varepsilon^{N-2}\right)}{\left(C(N, \alpha)^{\frac{N}{2}} S_{H, L}^{\frac{2 N-\alpha}{2}}-O\left(\varepsilon^{\frac{2 N-\alpha}{2}}\right)\right)^{\frac{N-2}{2 N-\alpha}}}\right)^{\frac{2 N-\alpha}{N+2-\alpha}}=\left(S_{H, L}\right)^{\frac{2 N-\alpha}{N+2-\alpha}} \cdot\left(\frac{1+O\left(\varepsilon^{N-2}\right)}{\left(1-O\left(\varepsilon^{\frac{2 N-\alpha}{2}}\right)\right)^{\frac{N-2}{2 N-\alpha}}}\right)^{\frac{2 N-\alpha}{N+2-\alpha}}
$$

and

$$
\left(\frac{1+O\left(\varepsilon^{N-2}\right)}{\left(1-O\left(\varepsilon^{\frac{2 N-\alpha}{2}}\right)\right)^{\frac{N-2}{2 N-\alpha}}}\right)^{\frac{2 N-\alpha}{N+2-\alpha}}<1+C(N, \alpha) \cdot \frac{O\left(\varepsilon^{N-2}\right)+O\left(\varepsilon^{\frac{2 N-\alpha}{2}}\right)}{\left(1-O\left(\varepsilon^{\frac{2 N-\alpha}{2}}\right)\right)^{\frac{N-2}{2 N-\alpha}}} .
$$

We observe that, for $\varepsilon>0$ sufficiently small, it holds

So,

$$
\left(1-O\left(\varepsilon^{\frac{N-2}{2 N-\alpha}}\right)\right)^{\frac{N-2}{2 N-\alpha}} \geq \frac{1}{2} .
$$

$$
\left(\frac{1+O\left(\varepsilon^{N-2}\right)}{\left(1-O\left(\varepsilon^{\frac{2 N-\alpha}{2}}\right)\right)^{\frac{N-2}{2 N-\alpha}}}\right)^{\frac{2 N-\alpha}{N+2-\alpha}}<1+2 C(N, \alpha)\left(O\left(\varepsilon^{N-2}\right)+O\left(\varepsilon^{\frac{2 N-\alpha}{2}}\right)\right)<1+O\left(\varepsilon^{\min \left\{N-2, \frac{2 N-\alpha}{2}\right\}}\right) .
$$

Therefore, we conclude that, for any $\varepsilon>0$ sufficiently small, we have

$$
\left(\frac{1}{\left(D\left(u_{\varepsilon}\right)\right)^{\frac{N-2}{2 N-\alpha}}} \int_{\mathbb{R}^{N}}\left|\nabla u_{\varepsilon}\right|^{2} \mathrm{~d} x\right)^{\frac{2 N-\alpha}{N+2-\alpha}}<\left(S_{H, L}\right)^{\frac{2 N-\alpha}{N+2-\alpha}}+O\left(\varepsilon^{\min \left\{N-2, \frac{2 N-\alpha}{2}\right\}}\right) .
$$


Combining (27) with (29), for $\varepsilon$ sufficiently small, we have

$$
\begin{aligned}
J_{A, V_{\mathcal{P}}}\left(t_{\varepsilon} u_{\varepsilon}\right)< & \frac{N+2-\alpha}{2(2 N-\alpha)}\left(S_{H, L}\right)^{\frac{2 N-\alpha}{N+2-\alpha}}+O\left(\varepsilon^{\min \left\{N-2, \frac{2 N-\alpha}{2}\right\}}\right) \\
& +\frac{1}{2}\left(\frac{1}{D\left(u_{\varepsilon}\right)^{\frac{N-2}{2 N-\alpha}}} \int_{\mathbb{R}^{N}}\left|\nabla u_{\varepsilon}\right|^{2} \mathrm{~d} x+\frac{1}{\left(D\left(u_{\varepsilon}\right)^{\frac{N-2}{2 N-\alpha}}\right.} \int_{\mathbb{R}^{N}}\left(|A(x)|^{2}+V_{\mathcal{P}}(x)\right)\left|u_{\varepsilon}\right|^{2} \mathrm{~d} x\right)^{\frac{N-2}{N+2-\alpha}} \\
& \cdot \frac{1}{\left(D\left(u_{\varepsilon}\right)^{\frac{N-2}{2 N-\alpha}}\right.} \int_{\mathbb{R}^{N}}\left(|A(x)|^{2}+V_{\mathcal{P}}(x)\right)\left|u_{\varepsilon}\right|^{2} \mathrm{~d} x-\frac{\lambda}{2 p} t_{\varepsilon}^{2 p} B\left(u_{\varepsilon}\right) .
\end{aligned}
$$

We claim that there is a positive constant $C_{0}$ such that, for all $\varepsilon>0$

$$
t_{\varepsilon}^{2 p} \geq C_{0} \text {. }
$$

In fact, suppose that there is a sequence $\left(\varepsilon_{n}\right) \subset \mathbb{R}, \varepsilon_{n} \rightarrow 0$ as $n \rightarrow \infty$, such that $t_{\varepsilon_{n}} \rightarrow 0$ as $n \rightarrow \infty$. Thus,

$$
0<c_{\lambda} \leq \sup _{t \geq 0} J_{A, V}\left(t u_{\varepsilon_{n}}\right)=J_{A, V_{\mathcal{P}}}\left(t_{\varepsilon_{n}} u_{\varepsilon_{n}}\right) .
$$

Since $u_{\varepsilon_{n}} \in H_{A, V_{\mathcal{P}}}^{1}\left(\mathbb{R}^{N}, \mathbb{C}\right)$ is bounded and $t_{\varepsilon_{n}} \rightarrow 0$, as $n \rightarrow \infty$, we have $t_{\varepsilon_{n}} u_{\varepsilon_{n}} \rightarrow 0$ as $n \rightarrow \infty$, em $H_{A, V_{\mathcal{P}}}^{1}\left(\mathbb{R}^{N}, \mathbb{C}\right)$.

The continuity of $J_{A, V_{\mathcal{P}}}$ implies that $J_{A, V_{\mathcal{P}}}\left(t_{\varepsilon_{n}} u_{\varepsilon_{n}}\right) \rightarrow J_{A, V_{\mathcal{P}}}(0)=0$. Therefore,

$$
0<c_{\lambda} \leq \lim _{n \rightarrow \infty} J_{A, V_{\mathcal{P}}}\left(t_{\varepsilon_{n}} u_{\varepsilon_{n}}\right)=0,
$$

a contradiction that proves the claim.

From (26), (30) and (31) we conclude that, for some constant $C_{0}>0$ and $\varepsilon>0$ sufficiently small we have

$$
\begin{aligned}
J_{A, V_{\mathcal{P}}}\left(t_{\varepsilon} u_{\varepsilon}\right)< & \frac{N+2-\alpha}{2(2 N-\alpha)}\left(S_{A}\right)^{\frac{2 N-\alpha}{N+2-\alpha}}+O\left(\varepsilon^{\min \left\{N-2, \frac{2 N-\alpha}{2}\right\}}\right) \\
& +\frac{1}{2}\left(\frac{1}{D\left(u_{\varepsilon}\right)^{\frac{N-2}{2 N-\alpha}}}\left\|u_{\varepsilon}\right\|_{A V_{\mathcal{P}}}^{2}\right)^{\frac{N-2}{N+2-\alpha}} \cdot \frac{1}{\left(D\left(u_{\varepsilon}\right)^{\frac{N-2}{2 N-\alpha}}\right.} \int_{\mathbb{R}^{N}}\left(|A(x)|^{2}+V_{\mathcal{P}}(x)\right)\left|u_{\varepsilon}\right|^{2} \mathrm{~d} x-C_{0} B\left(u_{\varepsilon}\right) \\
< & \frac{N+2-\alpha}{2(2 N-\alpha)}\left(S_{A}\right)^{\frac{2 N-\alpha}{N+2-\alpha}} \\
& +O\left(\varepsilon^{\min \left\{N-2, \frac{2 N-\alpha}{2}\right\}}\right)+\frac{S_{A}(\varepsilon)^{2}}{2} \cdot \int_{\mathbb{R}^{N}}\left(|A(x)|^{2}+V_{\mathcal{P}}(x)\right)\left|u_{\varepsilon}\right|^{2} \mathrm{~d} x-C_{0} B\left(u_{\varepsilon}\right) .
\end{aligned}
$$

Thus,

$$
J_{A, V_{\mathcal{P}}}\left(t_{\varepsilon} u_{\varepsilon}\right)<\frac{N+2-\alpha}{2(2 N-\alpha)}\left(S_{A}\right)^{\frac{2 N-\alpha}{N+2-\alpha}}+O\left(\varepsilon^{\eta}\right)+C_{1} \int_{\mathbb{R}^{N}} a(x)\left|u_{\varepsilon}\right|^{2} \mathrm{~d} x-C_{0} B\left(u_{\varepsilon}\right),
$$

where $C_{1}=\frac{S_{A}(\varepsilon)^{2}}{2}, a(x)=|A(x)|^{2}+V_{p}(x)$ and $\eta=\min \left\{N-2, \frac{2 N-\alpha}{2}\right\}$.

By direct computation we know that, for $\varepsilon<1$,

$$
\begin{aligned}
B\left(u_{\varepsilon}\right) & =\int_{\mathbb{R}^{N}} \int_{\mathbb{R}^{N}} \frac{\varepsilon^{\frac{(2-N) p}{2}}[N(N-2)]^{\frac{(N-2) p}{4}} \varepsilon^{\frac{(2-N) p}{2}}[N(N-2)]^{\frac{(N-2) p}{4}}}{\left(1+\left|\frac{x}{\varepsilon}\right|^{2}\right)^{\frac{(N-2) p}{2}}|x-y|^{\alpha}\left(1+\left|\frac{y}{\varepsilon}\right|^{2}\right)^{\frac{(N-2) p}{4}}} \mathrm{~d} x \mathrm{~d} y \\
& =[N(N-2)]^{\frac{(N-2) p}{2}} \varepsilon^{2 N-\alpha-(N-2) p} \int_{B_{\frac{\delta}{\varepsilon}}} \int_{B_{B^{\frac{\delta}{\varepsilon}}}} \frac{1}{\left(1+|x|^{2}\right)^{\frac{(N-2) p}{2}}|x-y|^{\alpha}\left(1+|y|^{2}\right)^{\frac{(N-2) p}{2}}} \mathrm{~d} x \mathrm{~d} y \\
& \geq[N(N-2)]^{\frac{(N-2) p}{2}} \varepsilon^{2 N-\alpha-(N-2) p} \int_{B_{\delta}} \int_{B_{\delta}} \frac{1}{\left(1+|x|^{2}\right)^{\frac{(N-2) p}{2}}|x-y|^{\alpha}\left(1+|y|^{2}\right)^{\frac{(N-2) p}{2}}} \mathrm{~d} x \mathrm{~d} y .
\end{aligned}
$$

Therefore,

$$
B\left(u_{\varepsilon}\right) \geq[N(N-2)]^{\frac{(N-2) p}{2}} \varepsilon^{2 N-\alpha-(N-2) p} \int_{B_{\delta}} \int_{B_{\delta}} \frac{1}{\left(1+|x|^{2}\right)^{\frac{(N-2) p}{2}}|x-y|^{\alpha}\left(1+|y|^{2}\right)^{\frac{(N-2) p}{2}}} \mathrm{~d} x \mathrm{~d} y .
$$


Since $a(x)$ is bounded, (33) and the last inequality imply that

$$
J_{A, V_{\mathcal{P}}}\left(t_{\varepsilon} u_{\varepsilon}\right)<\frac{N+2-\alpha}{2(N-\alpha)}\left(S_{A}\right)^{\frac{2 N-\alpha}{N+2-\alpha}}+O\left(\varepsilon^{\eta}\right)+C_{2} \int_{\mathbb{R}^{N}}\left|u_{\varepsilon}(x)\right|^{2} \mathrm{~d} x-C_{3} \varepsilon^{2 N-\alpha-(N-2) p} .
$$

We are going to show that

$$
\lim _{\varepsilon \rightarrow 0} \varepsilon^{-\eta}\left(C_{2} \int_{\mathbb{R}^{N}}\left|u_{\varepsilon}(x)\right|^{2} \mathrm{~d} x-C_{3} \varepsilon^{2 N-\alpha-(N-2) p}\right)=-\infty .
$$

In order to do that, it suffices to show that

$$
\lim _{\varepsilon \rightarrow 0} \varepsilon^{-\eta}\left(C_{2} \int_{B_{\delta}}\left|u_{\varepsilon}(x)\right|^{2} \mathrm{~d} x-C_{3} \varepsilon^{2 N-\alpha-(N-2) p}\right)=-\infty
$$

and

$$
C_{2} \int_{B_{2 \delta} \backslash B_{\delta}}\left|u_{\varepsilon}(x)\right|^{2} \mathrm{~d} x-C_{3} \varepsilon^{2 N-\alpha-(N-2) p}=O\left(\varepsilon^{\eta}\right) .
$$

Assuming (36) and (37), let us proceed with our proof. Since

$$
O\left(\varepsilon^{\eta}\right)+C_{2} \int_{\mathbb{R}^{N}}\left|u_{\varepsilon}\right|^{2} \mathrm{~d} x-C_{3} \varepsilon^{2 N-\alpha-(N-2) p}=\varepsilon^{\eta}\left[\frac{O\left(\varepsilon^{\eta}\right)}{\varepsilon^{\eta}}+\varepsilon^{-\eta}\left(C_{2} \int_{\mathbb{R}^{N}}\left|u_{\varepsilon}(x)\right|^{2} \mathrm{~d} x-C_{3} \varepsilon^{2 N-\alpha-(N-2) p}\right)\right],
$$

from (35) follows

$$
O\left(\varepsilon^{\eta}\right)+C_{2} \int_{\mathbb{R}^{N}}\left|u_{\varepsilon}(x)\right|^{2} \mathrm{~d} x-C_{3} \varepsilon^{2 N-\alpha-(N-2) p}<0
$$

for $\varepsilon>0$ sufficiently small.

Thus, (34) and (38) imply

$$
\sup _{t \geq 0} J_{A, V_{\mathcal{P}}}\left(t u_{\varepsilon}\right)<\frac{N+2-\alpha}{2(2 N-\alpha)}\left(S_{A}\right)^{\frac{2 N-\alpha}{N+2-\alpha}}
$$

for $\varepsilon>0$ sufficiently small and fixed. Once (36) and (37) are verified, the proof of Case 1 is complete.

We now prove (36).

Lemma 15. If $\frac{N+2-\alpha}{N-2}<p<2_{\alpha}^{*}$ and $N=3,4$ or $\frac{2 N-2-\alpha}{N-2}<p<2_{\alpha}^{*}$ and $N \geq 5$ it follows that

$$
\lim _{\varepsilon \rightarrow 0} \varepsilon^{-\eta}\left(C_{2} \int_{B_{\delta}}\left|u_{\varepsilon}(x)\right|^{2} \mathrm{~d} x-C_{3} \varepsilon^{2 N-\alpha-(N-2) p}\right)=-\infty
$$

Proof. This limit is evaluated considering the cases $N=3, N=4$ and $N \geq 5$ as follows. We initially observe that direct computation allows us to conclude that

$$
\int_{B_{\delta}}\left|u_{\varepsilon}(x)\right|^{2} \mathrm{~d} x=N \omega_{N}[N(N-2)]^{\frac{N-2}{2}} \varepsilon^{2} \int_{0}^{\frac{\delta}{\varepsilon}} \frac{r^{N-1}}{\left(1+r^{2}\right)^{N-2}} \mathrm{~d} r
$$

where $\omega_{N}$ denotes the volume of the unit ball in $\mathbb{R}^{N}$.

Now, define

$$
I_{\varepsilon}:=\varepsilon^{-\eta}\left(C_{2} \int_{B_{\delta}}\left|u_{\varepsilon}(x)\right|^{2} \mathrm{~d} x-C_{3} \varepsilon^{2 N-\alpha-(N-2) p}\right)=\varepsilon^{-\eta}\left(C_{4} \varepsilon^{2} \int_{0}^{\frac{\delta}{\varepsilon}} \frac{r^{N-1}}{\left(1+r^{2}\right)^{N-2}} \mathrm{~d} r-C_{3} \varepsilon^{2 N-\alpha-(N-2) p}\right),
$$

the second equality being a consequence of (39).

- The case $\mathbf{N}=\mathbf{3}$. In this case we have $5-\alpha<p<2_{\alpha}^{*}$ and therefore $5-\alpha-p<0$. We also observe that $0<\alpha<N$ implies $\min \left\{N-2, \frac{2 N-\alpha}{2}\right\}=N-2=1$.

It is easy to show that

$$
\varepsilon^{2} \int_{0}^{\frac{\delta}{\varepsilon}} \frac{r^{2}}{1+r^{2}} \mathrm{~d} r=\varepsilon\left(\delta-\varepsilon \arctan \left(\frac{\delta}{\varepsilon}\right)\right) .
$$


Thus,

$$
I_{\varepsilon}=C_{4}\left(\delta-\varepsilon \arctan \left(\frac{\delta}{\varepsilon}\right)\right)-C_{3} \varepsilon^{5-\alpha-p}
$$

Our claim follows.

- The case $\mathbf{N}=4$. In this case, $\frac{6-\alpha}{2}<p<2_{\alpha}^{*}$ implies $6-\alpha-2 p<0$ and $\min \left\{N-2, \frac{2 N-\alpha}{2}\right\}=N-2=2$, since $0<\alpha<4$.

Changing variables, we obtain

$$
\varepsilon^{2} \int_{0}^{\frac{\delta}{\varepsilon}} \frac{r^{3}}{\left(1+r^{2}\right)^{2}} \mathrm{~d} r=\frac{\varepsilon^{2}}{2}\left[\ln \left(1+\frac{\delta^{2}}{\varepsilon^{2}}\right)+\frac{\varepsilon^{2}}{\varepsilon^{2}+\delta^{2}}-1\right] .
$$

So,

$$
\begin{aligned}
I_{\varepsilon} & =\frac{C_{4}}{2}\left(\ln \left(1+\frac{\delta^{2}}{\varepsilon^{2}}\right)+\frac{\varepsilon^{2}}{\varepsilon^{2}+\delta^{2}}-1\right)-C_{3} \varepsilon^{6-\alpha-2 p} \\
& =\ln \left(1+\frac{\delta^{2}}{\varepsilon^{2}}\right)\left[\frac{C_{4}}{2}+\frac{C_{4}}{2 \ln \left(1+\frac{\delta^{2}}{\varepsilon^{2}}\right)} \frac{\varepsilon^{2}}{\varepsilon^{2}+\delta^{2}}-\frac{C_{4}}{2 \ln \left(1+\frac{\delta^{2}}{\varepsilon^{2}}\right)}-C_{3} \frac{\varepsilon^{6-\alpha-2 p}}{\ln \left(1+\frac{\delta^{2}}{\varepsilon^{2}}\right)}\right] .
\end{aligned}
$$

Our claim follows by applying L'Hospital rule.

- The case $\mathbf{N} \geq \mathbf{5}$. We have

$$
I_{\varepsilon}=\varepsilon^{2-\min \left\{N-2, \frac{2 N-\alpha}{2}\right\}}\left(C_{4} \int_{0}^{\frac{\delta}{\varepsilon}} \frac{r^{N-1}}{\left(1+r^{2}\right)^{N-2}} \mathrm{~d} r-C_{3} \varepsilon^{2 N-\alpha-(N-2) p-2}\right) .
$$

It is easy to show that, if $N \geq 5$, then the integral

converges.

$$
\lim _{\varepsilon \rightarrow 0} \int_{0}^{\frac{\delta}{\varepsilon}} \frac{r^{N-1}}{\left(1+r^{2}\right)^{N-2}} \mathrm{~d} r
$$

There are two cases to be considered:

- $0<\alpha<4$ and $N \geq 5$;

- $\alpha \geq 4$ and $N \geq 5$.

Let us suppose $0<\alpha<4$ and $N \geq 5$. Since $0<\alpha<4$ we have

$$
2-\eta=2-\min \left\{N-2, \frac{2 N-\alpha}{2}\right\}=-N+4<0 .
$$

Also $\frac{2 N-\alpha-2}{N-2}<p<\frac{2 N-\alpha}{N-2}$ implies $2 N-\alpha-(N-2) p-2<0$. Therefore, $I_{\varepsilon} \rightarrow-\infty$ as $\varepsilon \rightarrow 0$.

Now we consider the case $\alpha \geq 4$ and $N \geq 5$. We have $N-2 \geq \frac{2 N-\alpha}{2}$ and therefore

$$
2-\eta=2-\min \left\{N-2, \frac{2 N-\alpha}{2}\right\}=2-N+\frac{\alpha}{2}<0 .
$$

Since

$$
I_{\varepsilon}=\varepsilon^{2-N+\frac{\alpha}{2}}\left[C_{4} \int_{0}^{\frac{\delta}{\varepsilon}} \frac{r^{N-1}}{\left(1+r^{2}\right)^{N-2}} \mathrm{~d} r-C_{3} \varepsilon^{2 N-\alpha-(N-2) p-2}\right],
$$

we conclude that $I_{\varepsilon} \rightarrow-\infty$. We are done.

We now prove (37).

Lemma 16. It holds

$$
C_{2} \int_{B_{2 \delta} \backslash B_{\delta}}\left|u_{\varepsilon}(x)\right|^{2} \mathrm{~d} x-C_{3} \varepsilon^{2 N-\alpha-(N-2) p}=O\left(\varepsilon^{\eta}\right) .
$$


Proof. Fix $\delta>0$ sufficiently large so that $U_{\varepsilon}^{2}(x) \leq \varepsilon^{1+\eta}$ if $|x| \geq \delta$. Since

$$
\begin{aligned}
\frac{1}{\varepsilon^{\eta}}\left[C_{2} \int_{B_{2 \delta} \backslash B_{\delta}}\left|u_{\varepsilon}(x)\right|^{2} \mathrm{~d} x-C_{3} \varepsilon^{2 N-\alpha-(N-2) p}\right] & <\frac{C_{2}}{\varepsilon^{\eta}} \int_{B_{2 \delta} \backslash B_{\delta}} \psi^{2}(x) U_{\varepsilon}^{2}(x) \mathrm{d} x \leq C_{2} \varepsilon\|\psi\|_{2} \\
& \leq C_{1} \varepsilon\|\psi\|_{A, V_{\mathcal{P}}},
\end{aligned}
$$

our proof is complete.

Case 2. For $\lambda$ sufficiently large, $\frac{2 N-\alpha}{N}<p \leq \frac{N+2-\alpha}{N-2}$ and $N=3,4$ or $\frac{2 N-\alpha}{N}<p \leq \frac{2 N-2-\alpha}{N-2}$ and $N \geq 5$.

Proof of Case 2. Define $g_{\lambda}:[0,+\infty) \rightarrow \mathbb{R}$ by

$$
g_{\lambda}(t)=J_{A, V_{\mathcal{P}}}\left(t u_{\varepsilon}\right)=\frac{t^{2}}{2} \int_{\mathbb{R}^{N}}\left[\left|\nabla u_{\varepsilon}\right|^{2}+\left(|A(x)|^{2}+V_{\mathcal{P}}(x)\right)\left|u_{\varepsilon}\right|^{2}\right] \mathrm{d} x-\frac{\lambda}{2 p} t^{2 p} B\left(u_{\varepsilon}\right)-\frac{1}{2 \cdot 2_{\alpha}^{*}} t^{2 \cdot 2_{\alpha}^{*}} D\left(u_{\varepsilon}\right) .
$$

We already know that $\lim _{t \rightarrow+\infty} g_{\lambda}(t)=-\infty$ as $t \rightarrow+\infty$ and $\max _{t \geq 0} g_{\lambda}(t)$ is attained at some $t_{\lambda}>0$ satisfying

$$
t_{\lambda} \int_{\mathbb{R}^{N}}\left[\left|\nabla u_{\varepsilon}\right|^{2}+\left(|A(x)|^{2}+V_{\mathcal{P}}(x)\right)\left|u_{\varepsilon}\right|^{2}\right] \mathrm{d} x=\lambda t_{\lambda}^{2 p-1} B\left(u_{\varepsilon}\right)+t_{\lambda}^{2 \cdot 2_{\alpha}^{*}-1} D\left(u_{\varepsilon}\right),
$$

that is,

$$
\int_{\mathbb{R}^{N}}\left[\left|\nabla u_{\varepsilon}\right|^{2}+\left(|A(x)|^{2}+V_{\mathcal{P}}(x)\right)\left|u_{\varepsilon}\right|^{2}\right] \mathrm{d} x=\lambda t_{\lambda}^{2(p-1)} B\left(u_{\varepsilon}\right)+t_{\lambda}^{2\left(2_{\alpha}^{*}-1\right)} D\left(u_{\varepsilon}\right),
$$

since $g_{\lambda}^{\prime}\left(t_{\lambda}\right)=0$. Thus $t_{\lambda} \rightarrow 0$ as $\lambda \rightarrow+\infty$ and

$$
\begin{aligned}
\max _{t \geq 0} J_{A, V_{\mathcal{P}}}\left(t u_{\varepsilon}\right) & =\frac{t_{\lambda}{ }^{2}}{2} \int_{\mathbb{R}^{N}}\left[\left|\nabla u_{\varepsilon}(x)\right|^{2}+\left(|A(x)|^{2}+V_{\mathcal{P}}(x)\right)\left|u_{\varepsilon}(x)\right|^{2}\right] \mathrm{d} x-\frac{\lambda}{2 p} t_{\lambda}^{2 p} B\left(u_{\varepsilon}\right)-\frac{1}{2 \cdot 2_{\alpha}^{*}} t^{2 \cdot 2_{\alpha}^{*}} D\left(u_{\varepsilon}\right) \\
& <\frac{t_{\lambda}{ }^{2}}{2} \int_{\mathbb{R}^{N}}\left[\left|\nabla u_{\varepsilon}\right|^{2}+\left(|A(x)|^{2}+V_{\mathcal{P}}(x)\right)\left|u_{\varepsilon}(x)\right|^{2}\right] \mathrm{d} x .
\end{aligned}
$$

Since $t_{\lambda} \rightarrow 0$ as $\lambda \rightarrow+\infty$ and $\frac{N+2-\alpha}{2(N-\alpha)}\left(S_{A}\right)^{\frac{2 N-\alpha}{N+2-\alpha}}>0$, we conclude that

$$
\frac{t_{\lambda}^{2}}{2} \int_{\mathbb{R}^{N}}\left[\left|\nabla u_{\varepsilon}\right|^{2}+\left(|A(x)|^{2}+V_{\mathcal{P}}(x)\right)\left|u_{\varepsilon}(x)\right|^{2}\right] \mathrm{d} x<\frac{N+2-\alpha}{2(2 N-\alpha)}\left(S_{A}\right)^{\frac{2 N-\alpha}{N+2-\alpha}}
$$

for $\lambda>0$ sufficiently large.

Therefore,

for $\lambda>0$ sufficiently large.

$$
\sup _{t \geq 0} J_{A, V_{\mathcal{P}}}\left(t u_{\varepsilon}\right)<\frac{N+2-\alpha}{2(2 N-\alpha)}\left(S_{A}\right)^{\frac{2 N-\alpha}{N+2-\alpha}}
$$

3.2. The proof of Theorem 1. Some arguments of this proof were adapted from the articles [2, 25].

Maintaining the notation introduced in subsection 3.1 consider the energy functional $I_{A, V}: H_{A, V}^{1}\left(\mathbb{R}^{N}, \mathbb{C}\right) \rightarrow \mathbb{R}$ given by

$$
I_{A, V}(u)=\frac{1}{2}\|u\|_{A, V}^{2}-\frac{1}{2 \cdot 2_{\alpha}^{*}} D(u)-\frac{\lambda}{2 p} B(u) .
$$

We denote by $\mathcal{N}_{A, V}$ the Nehari Manifold related to $I_{A, V}$, that is,

$$
\mathcal{N}_{A, V}=\left\{u \in H_{A, V}^{1}\left(\mathbb{R}^{N}, \mathbb{C}\right) \backslash\{0\}:\|u\|_{A, V}^{2}=D(u)+\lambda B(u)\right\},
$$

which is non-empty as a consequence of Theorem 13, As before, the functional $I_{A, V}$ satisfies the mountain pass geometry. Thus, there exists a sequence $\left(u_{n}\right) \subset H_{A, V}^{1}\left(\mathbb{R}^{N}, \mathbb{C}\right)$ such that

$$
I_{A, V}^{\prime}\left(u_{n}\right) \rightarrow 0 \quad \text { and } \quad I_{A, V}\left(u_{n}\right) \rightarrow d_{\lambda},
$$


where $d_{\lambda}$ is the minimax level, also characterized by

$$
d_{\lambda}=\inf _{u \in H_{A, V}^{1}\left(\mathbb{R}^{N}, \mathbb{C}\right) \backslash\{0\}} \max _{t \geq 0} I_{A, V}(t u)=\inf _{\mathcal{N}_{A, V}} I_{A, V}(u)>0 .
$$

We stress that, as a consequence of $\left(V_{2}\right)$, we have $I_{A, V}(u)<J_{A, V_{\mathcal{P}}}(u)$ for all $u \in H_{A, V}^{1}\left(\mathbb{R}^{N}, \mathbb{C}\right)$.

The next lemma compares the levels $d_{\lambda}$ and $c_{\lambda}$.

Lemma 17. The levels $d_{\lambda}$ and $c_{\lambda}$ verify the inequality

$$
d_{\lambda}<c_{\lambda}<\frac{N+2-\alpha}{2(2 N-\alpha)}\left(S_{A}\right)^{\frac{2 N-\alpha}{N+2-\alpha}}
$$

for all $\lambda>0$.

Proof. Let $u$ be the ground state solution of problem (10) and consider $\bar{t}_{u}>0$ such that $\bar{t}_{u} u \in \mathcal{N}_{A, V}$, that is

$$
0<d_{\lambda} \leq \sup _{t \geq 0} I_{A, V}(t u)=I_{A, V}\left(\bar{t}_{u} u\right) .
$$

It follows from $\left(V_{2}\right)$ that

$$
0<d_{\lambda} \leq I_{A, V}\left(\bar{t}_{u} u\right)<J_{A, V_{\mathcal{P}}}\left(\bar{t}_{u} u\right) \leq \sup _{t \geq 0} J_{A, V_{\mathcal{P}}}(t u)=J_{A, V_{\mathcal{P}}}(u)=c_{\lambda} .
$$

Therefore,

$$
d_{\lambda}<c_{\lambda} .
$$

The second inequality was already known.

Proof of Theorem 1 Let $\left(u_{n}\right)$ be a $(P S)_{d_{\lambda}}$ sequence for $I_{A, V}$. As before, $\left(u_{n}\right)$ is bounded in $H_{A, V}^{1}\left(\mathbb{R}^{N}, \mathbb{C}\right)$. Thus, there exists $u \in H_{A, V}^{1}\left(\mathbb{R}^{N}, \mathbb{C}\right)$ such that

$$
u_{n} \rightarrow u \text { in } H_{A, V}^{1}\left(\mathbb{R}^{N}, \mathbb{C}\right) .
$$

By the same arguments given in the proof of Theorem 13 $u$ is a ground state solution of problem (3), if $u \neq 0$.

Following close [2], we will show that $u=0$ cannot occur. Indeed, Lemma 6 yields

$$
\lim _{n \rightarrow \infty} \int_{\mathbb{R}^{N}} W\left|u_{n}\right|^{2} \mathrm{~d} x=0,
$$

since $W \in L^{\frac{N}{2}}\left(\mathbb{R}^{N}, \mathbb{C}\right)$ and $u_{n} \rightarrow 0$ in $H_{A, V}^{1}\left(\mathbb{R}^{N}, \mathbb{C}\right)$. So,

$$
\left|J_{A, V_{\mathcal{P}}}\left(u_{n}\right)-I_{A, V}\left(u_{n}\right)\right|=o_{n}(1)
$$

showing that

$$
J_{A, V_{\mathcal{P}}}\left(u_{n}\right) \rightarrow d_{\lambda}
$$

But, for $\varphi \in H_{A, V}^{1}\left(\mathbb{R}^{N}, \mathbb{C}\right)$ such that $\|\varphi\|_{A, V} \leq 1$, we have

$$
\left|\left(J_{A, V_{\mathcal{P}}}^{\prime}\left(u_{n}\right)-I_{A, V}^{\prime}\left(u_{n}\right)\right) \cdot \varphi\right| \leq\left(\int_{\mathbb{R}^{N}} W\left|u_{n}\right|^{2} \mathrm{~d} x\right)^{\frac{1}{2}}=o_{n}(1) .
$$

Thus,

$$
J_{A, V_{\mathcal{P}}}^{\prime}\left(u_{n}\right)=o_{n}(1)
$$

Let $t_{n}>0$ such that $t_{n} u_{n} \in \mathcal{M}_{A, V_{\mathcal{P}}}$. Mimicking the argument found in [1, 19, 29, 31, it follows that $t_{n} \rightarrow 1$ as $n \rightarrow \infty$. Therefore,

Letting $n \rightarrow+\infty$, we get

$$
c_{\lambda} \leq J_{A, V_{\mathcal{P}}}\left(t_{n} u_{n}\right)=J_{A, V_{\mathcal{P}}}\left(u_{n}\right)+o_{n}(1)=d_{\lambda}+o_{n}(1) .
$$

$$
c_{\lambda} \leq d_{\lambda}
$$

obtaining a contradiction with Lemma 17. This completes the proof of Theorem 1 


\section{The CASE $f(u)=|u|^{p-1} u$}

4.1. The periodic problem. In this subsection we deal with problem (6) for $f(u)$ as above, that is,

$$
\left.-(\nabla+i A(x))^{2} u+V_{\mathcal{P}}(x) u=\left(\frac{1}{|x|^{\alpha}} *|u|^{2_{\alpha}^{*}}\right)\right)|u|^{2_{\alpha}^{*}-2} u+\lambda|u|^{p-1} u,
$$

where $1<p<2^{*}-1$.

We observe that in this case the energy functional $J_{A, V_{\mathcal{P}}}$ is given by

where, as before

$$
J_{A, V_{\mathcal{P}}}(u):=\frac{1}{2}\|u\|_{A, V_{\mathcal{P}}}^{2}-\frac{1}{2 \cdot 2_{\alpha}^{*}} D(u)-\frac{\lambda}{p+1} \int_{\mathbb{R}^{N}}|u|^{p+1} \mathrm{~d} x,
$$

$$
D(u)=\int_{\mathbb{R}^{N}}\left(\frac{1}{|x|^{\alpha}} *|u|^{2_{\alpha}^{*}}\right)|u|^{2_{\alpha}^{*}} \mathrm{~d} x=\int_{\mathbb{R}^{N}} \int_{\mathbb{R}^{N}} \frac{\left|u(x)^{2_{\alpha}^{*}}\right||u(y)|^{2_{\alpha}^{*}}}{|x-y|^{\alpha}} \mathrm{d} x \mathrm{~d} y .
$$

By the Sobolev immersion (7) and the Hardy-Littlewood-Sobolev inequality, we have that $J_{A, V_{\mathcal{P}}}$ is well defined.

Definition 4.1. A function $u \in H_{A, V_{\mathcal{P}}}^{1}\left(\mathbb{R}^{N}, \mathbb{C}\right)$ is a weak solution of (42) if

$$
\left.\langle u, \varphi\rangle_{A, V_{\mathcal{P}}}-\mathfrak{R e} \int_{\mathbb{R}^{N}}\left(\frac{1}{|x|^{\alpha}} *|u|^{2_{\alpha}^{*}}\right)\right)|u|^{2_{\alpha}^{*}-2} u \bar{\psi} \mathrm{d} x-\lambda \mathfrak{R e} \int_{\mathbb{R}^{N}}|u|^{p-1} u \bar{\psi} \mathrm{d} x=0
$$

for all $\psi \in H_{A, V_{\mathcal{P}}}^{1}\left(\mathbb{R}^{N}, \mathbb{C}\right)$.

As before, we see that critical points of $J_{A, V_{\mathcal{P}}}$ are weak solutions of (42) and

$$
J_{A, V_{\mathcal{P}}}^{\prime}(u) \cdot u:=\|u\|_{A, V_{\mathcal{P}}}^{2}-D(u)-\lambda\|u\|_{p+1}^{p+1} .
$$

We obtain that $J_{A, V_{\mathcal{P}}}$ satisfies the geometry of the mountain pass (see the proof of Lemma 7 ).

As in Section 3] the mountain pass theorem without the PS condition yields a sequence $\left(u_{n}\right) \subset H_{A, V_{\mathcal{P}}}^{1}\left(\mathbb{R}^{N}, \mathbb{C}\right)$ such that

$$
J_{A, V_{\mathcal{P}}}^{\prime}\left(u_{n}\right) \rightarrow 0 \quad \text { and } \quad J_{A, V_{\mathcal{P}}}\left(u_{n}\right) \rightarrow c_{\lambda},
$$

where $c_{\lambda}=\inf _{\alpha \in \Gamma} \max _{t \in[0,1]} J_{A, V_{\mathcal{P}}}(\gamma(t))$ and $\Gamma=\left\{\gamma \in C^{1}\left([0,1], H_{A, V_{\mathcal{P}}}^{1}\left(\mathbb{R}^{N}, \mathbb{C}\right)\right): \gamma(0)=0, J_{A, V_{\mathcal{P}}}(\gamma(1))<0\right\}$.

Considering the Nehari manifold $J_{A, V_{\mathcal{P}}}$

$$
\mathcal{M}_{A, V_{\mathcal{P}}}=\left\{u \in H_{A, V_{\mathcal{P}}}^{1}\left(\mathbb{R}^{N}, \mathbb{C}\right) \backslash\{0\}:\|u\|_{A, V_{\mathcal{P}}}^{2}=D(u)+\lambda\|u\|_{p+1}^{p+1}\right\},
$$

by proceeding as in the proof of Lemma 11 we obtain

Lemma 18. There exists a unique $t_{u}=t_{u}(u)>0$ such that $t_{u} u \in \mathcal{M}_{A, V_{\mathcal{P}}}$ for all $u \in H_{A, V_{\mathcal{P}}}^{1}\left(\mathbb{R}^{N}, \mathbb{C}\right) \backslash\{0\}$ and $J_{A, V_{\mathcal{P}}}\left(t_{u} u\right)=\max _{t \geq 0} J_{A, V_{\mathcal{P}}}(t u)$. Moreover $c_{\lambda}=c_{\lambda}^{*}=c_{\lambda}^{* *}$, where

$$
c_{\lambda}^{*}=\inf _{u \in \mathcal{M}_{A, V_{\mathcal{P}}}} J_{A, V_{\mathcal{P}}}(u) \quad \text { and } \quad c_{\lambda}^{* *}=\inf _{u \in H_{A, V_{\mathcal{P}}}^{1}\left(\mathbb{R}^{N}, \mathbb{C}\right) \backslash\{0\}} \max _{t \geq 0} J_{A, V_{\mathcal{P}}}(t u) .
$$

Lemma 19. Suppose that $u_{n} \rightarrow u_{0}$ and consider

$$
B^{\prime}\left(u_{n}\right) \cdot \psi=\mathfrak{R e} \int_{\mathbb{R}^{N}}|u|^{p-1} u \bar{\psi}
$$

and

$$
\left.D^{\prime}\left(u_{n}\right) \cdot \psi=\mathfrak{R e} \int_{\mathbb{R}^{N}}\left(\frac{1}{|x|^{\alpha}} *\left|u_{n}\right|^{2_{\alpha}^{*}}\right)\right)\left|u_{n}\right|^{2_{\alpha}^{*}-2} u_{n} \bar{\psi}
$$

for $\psi \in C_{c}^{\infty}\left(\mathbb{R}^{N}, \mathbb{C}\right)$. Then $B^{\prime}\left(u_{n}\right) \cdot \psi \rightarrow B^{\prime}\left(u_{0}\right) \cdot \psi$ and $D^{\prime}\left(u_{n}\right) \cdot \psi \rightarrow D^{\prime}\left(u_{0}\right) \cdot \psi$ as $n \rightarrow \infty$.

Lemma 20. If $\left(u_{n}\right) \subset H_{A, V_{\mathcal{P}}}^{1}\left(\mathbb{R}^{N}, \mathbb{C}\right)$ is a $(P S)_{\lambda}$ sequence for $J_{A, V_{\mathcal{P}}}$, then $\left(u_{n}\right)$ is bounded. In addition, if $u_{n} \rightarrow u$ weakly in $H_{A, V_{\mathcal{P}}}^{1}\left(\mathbb{R}^{N}, \mathbb{C}\right)$, as $n \rightarrow \infty$, then $u$ is ground state solution for problem (42). 
Lemma 21. If $\left(u_{n}\right) \subset H_{A, V_{\mathcal{P}}}^{1}\left(\mathbb{R}^{N}, \mathbb{C}\right)$ is a sequence $(P S)_{c_{\lambda}}$ for $J_{A, V_{\mathcal{P}}}$ such that

$$
u_{n} \rightarrow 0 \text { weakly in } H_{A, V_{\mathcal{P}}}^{1}\left(\mathbb{R}^{N}, \mathbb{C}\right) \text { as } n \rightarrow \infty \text {, }
$$

with

$$
c_{\lambda}<\frac{N+2-\alpha}{2(2 N-\alpha)} S_{A}^{\frac{2 N-\alpha}{N+2-\alpha}}
$$

then there exists a sequence $\left(y_{n}\right) \in \mathbb{R}^{N}$ and constants $R, \theta>0$ such that

$$
\limsup _{n \rightarrow \infty} \int_{B_{r}\left(y_{n}\right)}\left|u_{n}\right|^{2} \mathrm{~d} x \geq \theta
$$

where $B_{r}(y)$ denotes the ball in $\mathbb{R}^{N}$ of center at $y$ and radius $r>0$.

The proof of Lemmas 19, 20 and 21 is similar to that of Corollary 9 Lemmas 10 and 12 respectively.

Lemma 22. Let $1<p<2^{*}-1$ and $u_{\varepsilon}$ as defined in (23). Then, there exists $\varepsilon$ such that

provided that either

$$
\sup _{t \geq 0} J_{A, V_{\mathcal{P}}}\left(t u_{\varepsilon}\right)<\frac{N+2-\alpha}{2(2 N-\alpha)}\left(S_{A}\right)^{\frac{2 N-\alpha}{N+2-\alpha}} \text {. }
$$

(i) $3<p<5, N=3$ and $\lambda>0$;

(ii) $p>1, N \geq 4$ and $\lambda>0$;

(iii) $1<p \leq 3, N=3$ and $\lambda$ sufficiently large.

Proof. Consider, for the cases $(i)$ and $(i i)$ the function $f:[0,+\infty) \rightarrow \mathbb{R}$ defined by

$$
f(t)=J_{A, V_{\mathcal{P}}}\left(t u_{\varepsilon}\right)=\frac{t^{2}}{2}\left\|u_{\varepsilon}\right\|_{A, V_{\mathcal{P}}}^{2}-\frac{t^{2 \cdot 2_{\alpha}^{*}}}{2 \cdot 2_{\alpha}^{*}} D\left(u_{\varepsilon}\right)-\frac{\lambda t^{p+1}}{p+1}\left\|u_{\varepsilon}\right\|_{p+1}^{p+1}
$$

and proceed as in the proof of Case 1, Lemma 14 .

In the case of $1<p \leq 3, N=3$ and $\lambda$ sufficiently large, consider $g_{\lambda}:[0,+\infty) \rightarrow \mathbb{R}$ defined by

$$
g_{\lambda}(t)=J_{A, V_{\mathcal{P}}}\left(t u_{\varepsilon}\right)=\frac{t^{2}}{2} \int_{\mathbb{R}^{N}}\left[\left|\nabla u_{\varepsilon}\right|^{2}+\left(|A(x)|^{2}+V_{\mathcal{P}}(x)\right)\left|u_{\varepsilon}\right|^{2}\right] \mathrm{d} x-\frac{1}{2 \cdot 2_{\alpha}^{*}} t^{2 \cdot 2_{\alpha}^{*}} D\left(u_{\varepsilon}\right)-\frac{\lambda t^{p+1}}{p+1}\left\|u_{\varepsilon}\right\|_{p+1}^{p+1}
$$

and proceed as in the proof of Case 2, Lemma 14 .

Similar to the proof of Theorem 13, we now state our result about the periodic problem (42).

Theorem 23. Under the hypotheses already stated on $A$ and $\alpha$, suppose that $\left(V_{1}\right)$ is valid. Then problem (42) has at least one ground state solution if either

(i) $3<p<5, N=3$ and $\lambda>0$;

(ii) $p>1, N \geq 4$ and $\lambda>0$;

(iii) $1<p \leq 3, N=3$ and $\lambda$ sufficiently large.

4.2. Proof of Theorem 2. Some arguments of this proof were adapted from the proof of Theorem 1 below, that in turn were adapted from articles [2, 25].

Maintaining the notation already introduced, consider the functional $I_{A, V}: H_{A, V}^{1}\left(\mathbb{R}^{N}, \mathbb{C}\right) \rightarrow \mathbb{R}$ defined by

$$
I_{A, V}(u):=\frac{1}{2}\|u\|_{A, V}^{2}-\frac{1}{2 \cdot 2_{\alpha}^{*}} D(u)-\frac{\lambda}{p+1}\|u\|_{p+1}^{p+1}
$$

for all $u \in H_{A, V}^{1}\left(\mathbb{R}^{N}, \mathbb{C}\right)$.

We denote by $\mathcal{N}_{A, V}$ the Nehari Manifold related to $I_{A, V}$, that is,

$$
\mathcal{N}_{A, V}=\left\{u \in H_{A, V}^{1}\left(\mathbb{R}^{N}, \mathbb{C}\right) \backslash\{0\}:\|u\|_{A, V}^{2}=D(u)+\lambda\|u\|_{p+1}^{p+1}\right\},
$$

which is non-empty as a consequence of Theorem 23. As before, the functional $I_{A, V}$ satisfies the mountain pass geometry. Thus, there exists a $(P S)_{d_{\lambda}}$ sequence $\left(u_{n}\right) \subset H_{A, V}^{1}\left(\mathbb{R}^{N}, \mathbb{C}\right)$, that is, a sequence satisfying

$$
I_{A, V}^{\prime}\left(u_{n}\right) \rightarrow 0 \quad \text { and } \quad I_{A, V}\left(u_{n}\right) \rightarrow d_{\lambda},
$$


where $d_{\lambda}$ is the minimax level, also characterized by

$$
d_{\lambda}=\inf _{u \in H_{A, V}^{1}\left(\mathbb{R}^{N}, \mathbb{C}\right) \backslash\{0\}} \max _{t \geq 0} I_{A, V}(t u)=\inf _{\mathcal{N}_{A, V}} I_{A, V}(u)>0 .
$$

As in the Section 3 , we have $I_{A, V}(u)<J_{A, V_{\mathcal{P}}}(u)$ for all $u \in H_{A, V}^{1}\left(\mathbb{R}^{N}, \mathbb{C}\right)$ as a consequence of $\left(V_{2}\right)$.

Similar to the proof of Lemma 17 we have the following conclusion that shows as important inequality involving the levels $d_{\lambda}$ and $c_{\lambda}$, what completes the proof of Theorem 2

Lemma 24. The levels $d_{\lambda}$ and $c_{\lambda}$ verify the inequality

$$
d_{\lambda}<c_{\lambda}<\frac{N+2-\alpha}{2(N-\alpha)}\left(S_{A}\right)^{\frac{2 N-\alpha}{N+2-\alpha}}
$$

for all $\lambda>0$.

$$
\text { 5. The CASE } f(u)=|u|^{2^{*}-2} u
$$

5.1. Proof of Theorem 3. As observed by Gao and Yang [20], the proof of Theorem 3 is analogous to the proof of Theorem 1 The principal distinction is that the $(P S)_{c_{\lambda}}$ condition holds true below the level $\frac{1}{N} S^{\frac{N}{2}}$. It follows from [31, Lemma 1.46] that

$$
\int_{\mathbb{R}^{N}}\left|\nabla u_{\varepsilon}\right|^{2} \mathrm{~d} x=S^{\frac{N}{2}}+O\left(\varepsilon^{N-2}\right)
$$

and

So, we have

$$
\int_{\mathbb{R}^{N}}\left|u_{\varepsilon}\right|^{2^{*}} \mathrm{~d} x=S^{\frac{N}{2}}+O\left(\varepsilon^{N}\right) .
$$

$$
\sup _{t \geq 0} J_{A, V_{\mathcal{P}}}\left(t_{\varepsilon} u_{\varepsilon}\right)<\frac{1}{N} S^{\frac{N}{2}}+O\left(\varepsilon^{N-2}\right)+C_{2} \int_{\mathbb{R}^{N}}\left|u_{\varepsilon}(x)\right|^{2} \mathrm{~d} x-C_{3} \varepsilon^{2 N-\alpha-(N-2) p}<\frac{1}{N} S^{\frac{N}{2}},
$$

since

$$
\lim _{\varepsilon \rightarrow 0} \varepsilon^{-(N-2)}\left(C_{2} \int_{\mathbb{R}^{N}}\left|u_{\varepsilon}(x)\right|^{2} \mathrm{~d} x-C_{3} \varepsilon^{2 N-\alpha-(N-2) p}\right)=-\infty .
$$

Observe that the last result is a consequence of

and

$$
\lim _{\varepsilon \rightarrow 0} \varepsilon^{-(N-2)}\left(C_{2} \int_{B_{\delta}}\left|u_{\varepsilon}(x)\right|^{2} \mathrm{~d} x-C_{3} \varepsilon^{2 N-\alpha-(N-2) p}\right)=-\infty
$$

The rest of the proof is omitted here.

$$
C_{2} \int_{B_{2 \delta} \backslash B_{\delta}}\left|u_{\varepsilon}(x)\right|^{2} \mathrm{~d} x-C_{3} \varepsilon^{2 N-\alpha-(N-2) p}=O\left(\varepsilon^{N-2}\right) .
$$

Acknowledgements. The authors thank Prof. G. M. Figueiredo for many useful conversations and suggestions.

\section{REFERENCES}

[1] C.O. Alves, P.C. Carrião, O.H. Miyagaki: Nonlinear perturbations of a periodic elliptic problem with critical growth, J. Math. Anal. Appl. 260 (2001), no. 1, 133-146.

[2] C.O. Alves and G.M. Figueiredo: Nonlinear perturbations of a periodic Kirchhoff equation in $\mathbb{R}^{N}$, Nonlinear Anal. 75 (2012), no. $5,2750-2759$.

[3] C.O. Alves, G.M. Figueiredo and M. Yang: Multiple semiclassical solutions for a nonlinear Choquard equation with magnetic field, Asymptot. Anal. 96 (2016), no. 2, 135-159.

[4] C.O. Alves, F. Gao, M. Squassina and M. Yang: Singularly perturbed critical Choquard equations, J. Differential Equations 263 (2017), no. 7, 3943-3988.

[5] C.O. Alves, A. Nobrega and M. Yang: Multi-bump solutions for Choquard equation with deepening potential well, Calc. Var. Partial Differential Equations 55 (2016), no. 3, Art. 48, 28 pp.

[6] C.O. Alves and M. Yang: Existence of semiclassical ground state solutions for a generalized Choquard equation, J. Differential Equations 257 (2014), no. 11, 4133-4164.

[7] C.O. Alves and M. Yang: Investigating the multiplicity and concentration behaviour of solutions for a quasi-linear Choquard equation via the penalization method, Proc. Roy. Soc. Edinburgh Sect. A 146 (2016), no. 1, 23-58. 
[8] V. Ambrosio and P. D'Avenia: Nonlinear fractional magnetic Schrödinger equation: existence and multiplicity, J. Differential Equations 264 (2018), no. 5, 3336-3368.

[9] V. Ambrosio: Concentration phenomena for a fractional Choquard equation with magnetic field, Dyn. Partial Differ. Equ. 16 (2019), no. 2, 125-149.

[10] G. Arioli and A. Szulkin: A semilinear Schrödinger equation in the presence of a magnetic field, Arch. Rational Mech. Anal. 170 (2003), no. 4, 277-295.

[11] H. Brézis and L. Nirenberg: Positive solutions of nonlinear elliptic equations involving critical Sobolev exponents, Comm. Pure Appl. Math 36 (1983), no. 4, 437-477.

[12] H. Bueno, G.G. Mamami and G.A. Pereira: Ground state of a magnetic nonlinear Choquard equation, Nonlinear Anal. 181 (2019), 189-199.

[13] S. Cingolani, M. Clapp and S. Secchi: Multiple solutions to a magnetic nonlinear Choquard equation, Z. Angew. Math. Phys. 63 (2012), no. 2, 233-248

[14] V. Coti Zelati and P.H. Rabinowtiz, Homoclinic Type Solutions for a Semilinear Elliptic PDE on $\mathbb{R}^{N}$, Comm. Pure Appl. Math. 45 (1992), no. 10, 1217-1269.

[15] P. d'Avenia and C. Ji: Multiplicity and concentration results for a magnetic Schrödinger equation with exponential critical growth in $\mathbb{R}^{2}$, arXiv:1906.1093\%.

[16] P. d'Avenia and M. Squassina: Ground states for fractional magnetic operators, ESAIM Control Optim. Calc. Var. 24 (2018), no. $1,1-24$.

[17] L. Du and M. Yang: Uniqueness and nondegeneracy of solutions for a critical nonlocal equation, Discrete Contin. Dyn. Syst. 39 (2019), no. 10, 5847-5866.

[18] L. Du, F. Gao and M. Yang: Existence and qualitative analysis for nonlinear weighted Choquard equations, arXiv:1810.11759v1.

[19] P. Felmer, A. Quaas and J. Tan: Positive solutions of the nonlinear Schrödinger equation with the fractional Laplacian, Proc. Roy. Soc. Edinburgh Sect. A 142 (2012), no. 6, 1237-1262.

[20] F. Gao and M. Yang: On nonlocal Choquard equations with Hardy-Littlewood-Sobolev critical exponents, J. Math. Anal. Appl. 448 (2017), no. 2, 1006-1041.

[21] F. Gao and M. Yang: The Brezis-Nirenberg type critical problem for nonlinear Choquard equation, Sci. China Math. 61 (2018), no. 7, 1219-1242.

[22] F. Gao and M. Yang: A strongly indefinite Choquard equation with critical exponent due to the Hardy-Littlewood-Sobolev inequality, Commun. Contemp. Math. 20 (2018), no. 4, 1750037, 22 pp.

[23] O. Kavian: Introduction à la théorie des points critiques et applications aux problèmes elliptiques, Springer-Verlag, Berlin, 1993, ISBN: 2-287-00410-6.

[24] E.H. Lieb and M. Loss: Analysis, 2nd. edition, Graduate Studies in Mathematics, vol. 14, American Mathematical Society, Providence, RI, 2001, ISBN: 0-8218-2783-9.

[25] O.H. Miyagaki: On a class of semilinear elliptic problem in $\mathbb{R}^{N}$ with critical growth, Nonlinear Anal. 29 (1997), no. 7, 773-781.

[26] V. Moroz and J. Van Schaftingen: Semi-classical states for the Choquard equation, Calc. Var. Partial Differential Equations 52 (2015), no. 1-2, 199-235.

[27] V. Moroz and J. Van Schaftingen: A guide to the Choquard equation, J. Fixed Point Theory Appl. 19 (2017), no. 1, 773-813.

[28] T. Mukherjee and K. Sreenadh: On concentration of least energy solutions for magnetic critical Choquard equations, J. Math. Anal. Appl. 464 (2018), no. 1, 402-420.

[29] P. H. Rabinowitz: On a class of nonlinear Schrödinger equations, Z. Angew. Math. Phys. 43 (1992), no. 2, $270-291$.

[30] Z. Shen, F. Gao and M. Yang: Multiple solutions for nonhomogeneous Choquard equation involving Hardy-Littlewood-Sobolev critical exponent, Z. Angew. Math. Phys. 68 (2017), no. 3, Art. 61, 25 pp.

[31] M. Willem: Minimax Theorems, Progress in Nonlinear Differential Equations and their Applications, 24. Birkhäuser Boston, Inc., Boston, MA, 1996, ISBN: 0-8176-3913-6. 